\title{
New series in the Johnson cokernels of the mapping class groups of surfaces
}

\author{
NAOYA ENOMOTO \\ TAKAO SATOH
}

Let $\Sigma_{g, 1}$ be a compact oriented surface of genus $g$ with one boundary component, and $\mathcal{M}_{g, 1}$ its mapping class group. Morita showed that the image of the $k^{\text {th }}$ Johnson homomorphism $\tau_{k}^{\mathcal{M}}$ of $\mathcal{M}_{g, 1}$ is contained in the kernel $\mathfrak{h}_{g, 1}(k)$ of an Sp-equivariant surjective homomorphism $H \otimes_{\mathbb{Z}} \mathcal{L}_{2 g}(k+1) \rightarrow \mathcal{L}_{2 g}(k+2)$, where $H:=H_{1}\left(\Sigma_{g, 1}, \mathbb{Z}\right)$ and $\mathcal{L}_{2 g}(k)$ is the degree $k$ part of the free Lie algebra $\mathcal{L}_{2 g}$ generated by $H$.

In this paper, we study the $\mathrm{Sp}$-module structure of the cokernel $\mathfrak{h}_{g, 1}^{\mathbb{Q}}(k) / \operatorname{Im}\left(\tau_{k, \mathbb{Q}}^{\mathcal{M}}\right)$ of the rational Johnson homomorphism $\tau_{k, \mathbb{Q}}^{\mathcal{M}}:=\tau_{k}^{\mathcal{M}} \otimes \mathrm{id}_{\mathbb{Q}}$, where $\mathfrak{h}_{g, 1}^{\mathbb{Q}}(k):=$ $\mathfrak{h}_{g, 1}(k) \otimes_{\mathbb{Z}} \mathbb{Q}$. In particular, we show that the irreducible Sp-module corresponding to a partition $\left[1^{k}\right]$ appears in the $k^{\text {th }}$ Johnson cokernel for any $k \equiv 1(\bmod 4)$ and $k \geq 5$ with multiplicity one. We also give a new proof of the fact due to Morita that the irreducible $\mathrm{Sp}$-module corresponding to a partition $[k]$ appears in the Johnson cokernel with multiplicity one for odd $k \geq 3$.

The strategy of the paper is to give explicit descriptions of maximal vectors with highest weight $\left[1^{k}\right]$ and $[k]$ in the Johnson cokernel. Our construction is inspired by the Brauer-Schur-Weyl duality between $\operatorname{Sp}(2 g, \mathbb{Q})$ and the Brauer algebras, and our previous work for the Johnson cokernel of the automorphism group of a free group.

20G05; 57M50

Dedicated to the memory of Midori Kato

\section{Introduction}

Dennis Johnson established a new remarkable method to investigate the group structure of the mapping class group of a surface and the Torelli group in a series of his pioneer works $[11 ; 12 ; 13 ; 14]$ in 1980 s. Especially, he gave a finite set of generators of the Torelli group, and constructed a homomorphism $\tau$ to determine the abelianization of the Torelli group. Now, his homomorphism $\tau$ is called the first Johnson homomorphism, and it is generalized to the Johnson homomorphisms of higher degrees. Over the last two decades, the study of the Johnson homomorphisms of the mapping class group has achieved a good progress by many authors including Morita [22], Hain [8] and so on. 
To put it plainly, the Johnson homomorphisms are used to describe "one by one approximations" of the Torelli group as follows. To explain it, let us fix some notation. For a compact oriented surface $\Sigma_{g, 1}$ of genus $g$ with one boundary component, let $\mathcal{M}_{g, 1}$ be its mapping class group. Namely, $\mathcal{M}_{g, 1}$ is a group of isotopy classes of orientation-preserving diffeomorphisms of $\Sigma_{g, 1}$ that fix the boundary component pointwise. The fundamental group $\pi_{1}\left(\Sigma_{g, 1}, *\right)$ of $\Sigma_{g, 1}$ is isomorphic to a free group $F_{2 g}$ of rank $2 g$. In this paper we fix an isomorphism $\pi_{1}\left(\Sigma_{g, 1}, *\right) \cong F_{2 g}$. Let $\Gamma_{2 g}(k)$ be the lower central series of $F_{2 g}$ beginning with $\Gamma_{2 g}(1)=F_{2 g}$, and set $\mathcal{L}_{2 g}(k):=\Gamma_{2 g}(k) / \Gamma_{2 g}(k+1)$. For each $k \geq 1$ let $\mathcal{M}_{g, 1}(k)$ be a normal subgroup of $\mathcal{M}_{g, 1}$ consisting of elements that act on $F_{2 g} / \Gamma_{2 g}(k+1)$ trivially. Then we have a descending filtration

$$
\mathcal{M}_{g, 1}(1) \supset \mathcal{M}_{g, 1}(2) \supset \cdots \supset \mathcal{M}_{g, 1}(k) \supset \cdots
$$

of $\mathcal{M}_{g, 1}$ such that the first term $\mathcal{M}_{g, 1}(1)$ is just the Torelli group $\mathcal{I}_{g, 1}$. This filtration is called the Johnson filtration of $\mathcal{M}_{g, 1}$. Set $\operatorname{gr}^{k}\left(\mathcal{M}_{g, 1}\right):=\mathcal{M}_{g, 1}(k) / \mathcal{M}_{g, 1}(k+1)$ for each $k \geq 1$. Then each of $\operatorname{gr}^{k}\left(\mathcal{M}_{g, 1}\right)$ is an $\operatorname{Sp}(2 g, \mathbb{Z})$-equivariant free abelian group of finite rank, and they are considered as one by one approximations of the Torelli group. Although to clarify the $\operatorname{Sp}(2 g, \mathbb{Z})$-module structure of each $\operatorname{gr}^{k}\left(\mathcal{M}_{g, 1}\right)$ plays an important role on various studies of the Torelli group; even to determine its rank is quite a difficult problem in general.

In order to study each graded quotients $\operatorname{gr}^{k}\left(\mathcal{M}_{g, 1}\right)$, the Johnson homomorphisms

$$
\tau_{k}^{\mathcal{M}}: \operatorname{gr}^{k}\left(\mathcal{M}_{g, 1}\right) \hookrightarrow H^{*} \otimes_{\mathbb{Z}} \mathcal{L}_{2 g}(k+1)
$$

of $\mathcal{M}_{g, 1}$ are valuable tools where $H:=H_{1}\left(\Sigma_{g, 1}, \mathbb{Z}\right)$ and $H^{*}:=\operatorname{Hom}_{\mathbb{Z}}(H, \mathbb{Z})$. Here we remark that $H^{*}$ is canonically isomorphic to $H$ by the Poincare duality. In general, the $k^{\text {th }}$ Johnson homomorphism is denoted by $\tau_{k}$ simply. In this paper, however, to distinguish the Johnson homomorphism of the mapping class group from that of the automorphism group of a free group, we attach a subscript $\mathcal{M}$ to that of the mapping class group. (See Section 3.3 for details.) Since each of $\tau_{k}^{\mathcal{M}}$ is an $\operatorname{Sp}(2 g, \mathbb{Z})$-equivariant injective homomorphism, determining the image $\operatorname{Im}\left(\tau_{k}^{\mathcal{M}}\right)$ of $\tau_{k}^{\mathcal{M}}$ is one of the most basic problems. In particular, from a representation-theoretic view, it is important to clarify the irreducible decomposition of $\operatorname{Im}\left(\tau_{k, \mathbb{Q}}^{\mathcal{M}}\right)$ as an $\operatorname{Sp}(2 g, \mathbb{Q})$-module where $\tau_{k, \mathbb{Q}}^{\mathcal{M}}:=\tau_{k}^{\mathcal{M}} \otimes \operatorname{id}_{\mathbb{Q}}$. In the following, the subscript $\mathbb{Q}$ always means tensoring with $\mathbb{Q}$ over $\mathbb{Z}$. Now, we have $\operatorname{Im}\left(\tau_{1}^{\mathcal{M}}\right) \cong \Lambda^{3} H$ due to Johnson [11]. Furthermore the $\operatorname{Sp}(2 g, \mathbb{Q})$-module structure of $\operatorname{Im}\left(\tau_{k, \mathbb{Q}}^{\mathcal{M}}\right)$ is completely determined for $1 \leq k \leq 4$. (See a table in Section 3.3.)

On the other hand, Morita [22] began to study the Johnson images systematically, and gave many remarkable results. Here we recall some of them. First, Morita [22] 
showed that $\operatorname{Im}\left(\tau_{k}^{\mathcal{M}}\right)$ is contained in the kernel $\mathfrak{h}_{g, 1}(k)$ of $H \otimes_{\mathbb{Z}} \mathcal{L}_{2 g}(k+1) \rightarrow$ $\mathcal{L}_{2 g}(k+2)$ for any $k \geq 2$. (See Section 3.3.) Second, he also showed that $\operatorname{Im}\left(\tau_{k}^{\mathcal{M}}\right)$ does not coincide with $\mathfrak{h}_{g, 1}(k)$ in general. Namely, the Johnson homomorphism $\tau_{k}^{\mathcal{M}}: \operatorname{gr}^{k}\left(\mathcal{M}_{g, 1}\right) \hookrightarrow \mathfrak{h}_{g, 1}(k)$ is not surjective in general. More precisely, he constructed an $\operatorname{Sp}(2 g, \mathbb{Q})$-equivariant surjective homomorphisms

$$
\operatorname{Tr}_{k}: \mathfrak{h}_{g, 1}^{\mathbb{Q}}(k) \rightarrow S^{k} H_{\mathbb{Q}}
$$

such that $\operatorname{Tr}_{k} \circ \tau_{k, \mathbb{Q}}^{\mathcal{M}} \equiv 0$ for any odd $k \geq 3$ using the Magnus representation of $\mathcal{M}_{g, 1}$. Here $S^{k} H_{\mathbb{Q}}$ is the symmetric tensor product of $H_{\mathbb{Q}}$ of degree $k$, and is isomorphic to the irreducible $\operatorname{Sp}(2 g, \mathbb{Q})$-module with highest weight $[k]$. Hence $S^{k} H_{\mathbb{Q}}$ appears in the irreducible decomposition of the cokernel

$$
\operatorname{Coker}\left(\tau_{k, \mathbb{Q}}^{\mathcal{M}}\right):=\mathfrak{h}_{g, 1}^{\mathbb{Q}}(k) / \operatorname{Im}\left(\tau_{k, \mathbb{Q}}^{\mathcal{M}}\right)
$$

for odd $k \geq 3$. We should remark that throughout the paper $\operatorname{Coker}\left(\tau_{k, \mathbb{Q}}^{\mathcal{M}}\right)$ denotes

$$
\mathfrak{h}_{g, 1}^{\mathbb{Q}}(k) / \operatorname{Im}\left(\tau_{k, \mathbb{Q}}^{\mathcal{M}}\right)
$$

not $H_{\mathbb{Q}}^{*} \otimes_{\mathbb{Q}} \mathcal{L}_{2 g}^{\mathbb{Q}}(k+1) / \operatorname{Im}\left(\tau_{k, \mathbb{Q}}^{\mathcal{M}}\right)$. Now, the map $\operatorname{Tr}_{k}$ is called the Morita trace, and $S^{k} H_{\mathbb{Q}}$ the Morita obstruction. Here the term "obstruction" means an obstruction for the surjectivity of the Johnson homomorphism $\tau_{k, \mathbb{Q}}^{\mathcal{M}}$.

From results for the irreducible decomposition of $\operatorname{Coker}\left(\tau_{k, \mathbb{Q}}^{\mathcal{M}}\right)$ for low degrees, it seems that the number of the irreducible components in $\operatorname{Coker}\left(\tau_{k, \mathbb{Q}}^{\mathcal{M}}\right)$ grows rapidly as degree increases. At the present stage, however, there are few results for obstructions other than the Morita obstruction for a general degree $k$. Thus, to establish a new method to detect a non-trivial irreducible component in $\operatorname{Coker}\left(\tau_{k}^{\mathcal{M}}\right)$ other than the Morita obstruction is an important problem in the study of the Johnson homomorphisms.

The main purpose of the paper is to detect new series of obstructions in the Johnson cokernels. To state our theorem, we will use the following notation. First, we remark that for each $k \geq 1$ the symmetric group $\mathfrak{S}_{k+2}$ of degree $k+2$ naturally acts on the space $H_{\mathbb{Q}}^{\otimes k+2}$ from the right as a permutation of the components. For each $1 \leq i \leq k+1$, denote by $s_{i} \in \mathfrak{S}_{k+2}$ the adjacent transposition between $i$ and $i+1$, and by $\sigma_{k+2}$ the cyclic permutation $s_{k+1} s_{k} \cdots s_{2} s_{1}$. Let $P$ be a subgroup of $\mathfrak{S}_{k+2}$ which fixes 1 . The group $P$ is isomorphic to $\mathfrak{S}_{k+1}$. The Dynkin-Specht-Wever element $\theta_{P}$ for $P$ in the group algebra $\mathbb{Q} \mathfrak{S}_{k+2}$ is defined to be

$$
\theta_{P}:=\left(1-s_{2}\right)\left(1-s_{3} s_{2}\right) \cdots\left(1-s_{k+1} s_{k} \cdots s_{2}\right) .
$$


Our main theorem is:

Theorem 1 (Theorem 7.8) Suppose $k \equiv 1(\bmod 4), k \geq 5$ and $g \geq k+2$. An element

$$
\varphi_{\left[1^{k}\right]}:=\left(\omega \otimes\left(e_{1} \wedge \cdots \wedge e_{k}\right)\right) \cdot \theta_{P} \cdot\left(1+\sigma_{k+2}+\cdots+\sigma_{k+2}^{k+1}\right)
$$

is an $\mathrm{Sp}$-maximal vector of weight $\left[1^{k}\right]$ in $\mathfrak{h}_{g, 1}^{\mathbb{Q}}(k)$. Moreover this gives a unique Sp-irreducible component with highest weight $\left[1^{k}\right]$ in $\operatorname{Coker} \tau_{k, \mathbb{Q}}^{\mathcal{M}}$.

In addition to this, we also give a new proof of the fact that the Morita obstruction uniquely appears in $\operatorname{Coker}\left(\tau_{k}^{\mathcal{M}}\right)$ for odd $k \geq 3$, due to Morita [22] and Nakamura. (See Theorem 7.7.)

In order to prove these, we use two key facts. The first one is a remarkable result with respect to $\operatorname{gr}^{k}\left(\mathcal{M}_{g, 1}\right)$ due to Hain [8]. In general, the graded $\operatorname{sum} \operatorname{gr}\left(\mathcal{M}_{g, 1}\right):=$ $\bigoplus_{k \geq 1} \operatorname{gr}^{k}\left(\mathcal{M}_{g, 1}\right)$ has a Lie algebra structure induced from the commutator bracket of $\mathcal{I}_{g, 1}$. In [8], Hain showed that the Lie algebra $\operatorname{gr}_{\mathbb{Q}}\left(\mathcal{M}_{g, 1}\right)$ is generated by the degree one part $\operatorname{gr}_{\mathbb{Q}}^{1}\left(\mathcal{M}_{g, 1}\right)$ as a Lie algebra. This shows the following. Let $\mathcal{M}_{g, 1}^{\prime}(k)$ be the lower central series of $\mathcal{I}_{g, 1}$ and set $\operatorname{gr}^{k}\left(\mathcal{M}_{g, 1}^{\prime}\right):=\mathcal{M}_{g, 1}^{\prime}(k) / \mathcal{M}_{g, 1}^{\prime}(k+1)$. Then we can define the Johnson homomorphism-like homomorphism

$$
\tau_{k}^{\prime \mathcal{M}}: \operatorname{gr}^{k}\left(\mathcal{M}_{g, 1}^{\prime}\right) \rightarrow \mathfrak{h}_{g, 1}(k) .
$$

(See Section 3.3.) Then Hain's result above induces $\operatorname{Im}\left(\tau_{k, \mathbb{Q}}^{\mathcal{M}}\right)=\operatorname{Im}\left(\tau_{k, \mathbb{Q}}^{\prime \mathcal{M}}\right)$ for any $k \geq 1$.

The second is our previous result for the cokernel of the Johnson homomorphism of the automorphism group of a free group. By a classical work of Dehn and Nielsen, it is known that a natural homomorphism $\mathcal{M}_{g, 1} \rightarrow \operatorname{Aut}\left(F_{2 g}\right)$ induced from the action of $\mathcal{M}_{g, 1}$ of the fundamental group $\pi_{1}\left(\Sigma_{g, 1}, *\right) \cong F_{2 g}$ is injective. Namely, we can consider $\mathcal{M}_{g, 1}$ as a subgroup of $\operatorname{Aut}\left(F_{2 g}\right)$. From this view point, we can apply results for the Johnson homomorphisms of $\operatorname{Aut}\left(F_{2 g}\right)$ to the study of that of $\mathcal{M}_{g, 1}$. For any $n \geq 2$, in general, a subgroup $\mathrm{IA}_{n}$ consisting of automorphisms of a free group $F_{n}$ that act on $H_{1}\left(F_{n}, \mathbb{Z}\right)$ trivially is called the IA-automorphism group of $F_{n}$. Let $\mathcal{A}_{n}^{\prime}(k)$ be the lower central series of $\mathrm{IA}_{n}$, and set $\operatorname{gr}^{k}\left(\mathcal{A}_{n}^{\prime}\right):=\mathcal{A}_{n}^{\prime}(k) / \mathcal{A}_{n}^{\prime}(k+1)$ for any $k \geq 1$. Then we can define the Johnson homomorphism $\tau_{k}^{\prime}: \operatorname{gr}^{k}\left(\mathcal{A}_{n}^{\prime}\right) \rightarrow H^{*} \otimes_{\mathbb{Z}} \mathcal{L}_{n}(k+1)$ for each $k \geq 1$. Then, in our paper [28], we showed that for $k \geq 2$ and $n \geq k+2$,

$$
\operatorname{Coker}\left(\tau_{k, \mathbb{Q}}^{\prime}\right) \cong \mathcal{C}_{n}^{\mathbb{Q}}(k),
$$

where $\mathcal{C}_{n}(k):=H^{\otimes k} /\left\langle a_{1} \otimes \cdots \otimes a_{k}-a_{2} \otimes \cdots \otimes a_{k} \otimes a_{1} \mid a_{i} \in H\right\rangle$. (See Section 3.3 for details.) 
In our previous paper [5], we gave the irreducible decomposition of $\operatorname{Coker}\left(\tau_{k, \mathbb{Q}}^{\prime}\right) \cong$ $\mathcal{C}_{n}^{\mathbb{Q}}(k)$ as a $\operatorname{GL}(n, \mathbb{Q})$-module. Especially, we showed that $S^{k} H_{\mathbb{Q}}$, which is also called the Morita obstruction, appears in $\operatorname{Coker}\left(\tau_{k, \mathbb{Q}}^{\prime}\right)$ with multiplicity one for any $k \geq 2$, and that $\Lambda^{k} H_{\mathbb{Q}}$ appears with multiplicity one for odd $k \geq 3$.

We remark that, as a $\operatorname{GL}(n, \mathbb{Q})-$ module, $\mathcal{C}_{n}^{\mathbb{Q}}(k)$ is isomorphic to the invariant part $a_{n}(k):=\left(H_{\mathbb{Q}}^{\otimes k}\right)^{\mathrm{Cyc}_{k}}$ of $H_{\mathbb{Q}}^{\otimes k}$ under the action of $\mathrm{Cyc}_{k}$. Namely, the cokernel $\operatorname{Coker}\left(\tau_{k, \mathbb{Q}}^{\prime}\right)$ is isomorphic to Kontsevich's $a_{n}(k)$ as a $\operatorname{GL}(n, \mathbb{Q})$-module. We also remark that in our notation $a_{n}(k)$ is considered for any $n \geq 2$ in contrast to Kontsevich's notation for even $n=2 g$. (See Kontsevich [17; 18].)

Combining Hain's result above with the fact $\operatorname{Coker}\left(\tau_{k, \mathbb{Q}}^{\prime}\right) \cong \mathcal{C}_{n}^{\mathbb{Q}}(k)$ for $n \geq k+2$, we can establish a new method to detect non-trivial $\mathrm{Sp}$-irreducible components in $\operatorname{Coker}\left(\tau_{k}^{\mathcal{M}}\right)$. (For more details, see Section 7.1.) The present paper produces the first successful results for the use of such method.

This paper is organized as follows. In Section 2, we fix some notation. In Section 3, we recall the theory of the Johnson homomorphisms for the mapping class groups of surfaces and the automorphism groups of free groups. Especially, we exposit Hain's remarkable result on the Johnson homomorphisms of the mapping class groups and the second author's result on those of the automorphism groups of free groups. In Section 4, we prepare some results from the highest weight theory for the symplectic group $\operatorname{Sp}(2 g, \mathbb{Q})$. By using Brauer-Schur-Weyl duality, we recall a description of the maximal vectors of $H_{\mathbb{Q}}^{\otimes k}$ as an $\operatorname{Sp}(2 g, \mathbb{Q})$-module. In Section 5, we explain a characterization of elements in $\mathfrak{h}_{g, 1}^{\mathbb{Q}}(k)$ by using the Dynkin-Specht-Wever idempotent and cyclic permutations. Combining this characterization with the description of maximal vectors in $H_{\mathbb{Q}}^{\otimes k}$ given in Section 4, we give a description of maximal vectors in $\mathfrak{h}_{g, 1}^{\mathbb{Q}}(k)$ in Section 7.3. In Section 6, by using Kraśkiewicz and Weyman's results, we give explicit calculations for the multiplicity of some irreducible representations of the cyclic group which are obtained from the restriction of those of the symmetric group. By using these, we obtain a multiplicity formula for some irreducible representation of $\operatorname{Sp}(2 g, \mathbb{Q})$ in $\mathfrak{h}_{g, 1}^{\mathbb{Q}}(k)$. This gives an upper bound on the multiplicities of Sp-irreducible representations in the Johnson cokernels.

Section 7 is the main chapter of this paper. First, we consider a new Sp-equivariant homomorphism $c_{k}: \mathfrak{h}_{g, 1}^{\mathbb{Q}}(k) \rightarrow \mathcal{C}_{2 g}^{\mathbb{Q}}(k)$. This $c_{k}$ is not injective and not surjective. Then, this gives a new class $\operatorname{Ker}\left(c_{k}\right)$ in the Johnson cokernels. Second, we give explicit multiplicities for Sp-irreducible modules $L_{\mathrm{Sp}}^{[k]}$ and $L_{\mathrm{Sp}}^{\left[1^{k}\right]}$ in $\mathfrak{h}_{g, 1}^{\mathbb{Q}}(k)$ and $\mathcal{C}_{2 g}^{\mathbb{Q}}(k)$. Third, we explicitly describe their maximal vectors. And we prove that they do not vanish by our $\mathrm{Sp}$-equivariant homomorphism $c_{k}$. Thus we detect a series of $\mathrm{Sp}$-irreducible components in the Johnson cokernels for the mapping class groups of surfaces. Finally, 
in Section 7.5, we discuss a gap between our $\operatorname{Ker}\left(c_{k}\right)$ and the $k^{\text {th }}$ Johnson image. Furthermore, we also give a problem on relationships between our results and Conant, Kassabov and Vogtmann's recent results on a structure of the abelianization of the graded Lie algebra $\mathfrak{h}_{g, 1}^{\mathbb{Q}}$.

Note added After we wrote this paper, Professor Hiroaki Nakamura told us about the following personal communication. In 1996, in his letter to Professor Shigeyuki Morita, he mentioned that, for $k=5,9,13$, an Sp-module $\left[1^{k}\right]$ appears in $\mathfrak{h}_{g, 1}^{\mathbb{Q}}(k)$ with multiplicity one, based on his explicit calculation in [24]. And he conjectured that these $\mathrm{Sp}$-irreducible components $\left[1^{k}\right]$ survive in the Johnson cokernel for $k \geq 5$ such that $k \equiv 1(\bmod 4)$.

\section{Notation}

Throughout the paper, we use the following notation. Let $G$ be a group and $N$ a normal subgroup of $G$.

- The binomial coefficient $\left(\begin{array}{l}n \\ r\end{array}\right)$ is denoted by ${ }_{n} C_{r}$.

- For any real number $x$, we set $\lfloor x\rfloor:=\max \{n \in \mathbb{Z} \mid n \leq x\}$.

- For any integer $p$, set

$$
\delta_{p \equiv a(\bmod m)}:= \begin{cases}1 & \text { if } p \equiv a(\bmod m) \\ 0 & \text { otherwise. }\end{cases}
$$

- The automorphism group $\operatorname{Aut}\left(F_{n}\right)$ of $F_{n}$ acts on $F_{n}$ from the right unless otherwise noted. For any $\sigma \in \operatorname{Aut}\left(F_{n}\right)$ and $x \in F_{n}$, the action of $\sigma$ on $x$ is denoted by $x^{\sigma}$.

- For an element $g \in G$, we also denote the coset class of $g$ by $g \in G / N$ if there is no confusion.

- For elements $x$ and $y$ of $G$, the commutator bracket $[x, y]$ of $x$ and $y$ is defined to be $[x, y]:=x y x^{-1} y^{-1}$.

- For elements $g_{1}, \ldots, g_{k} \in G$, a left-normed commutator

$$
\left[\left[\cdots\left[\left[g_{1}, g_{2}\right], g_{3}\right], \cdots\right], g_{k}\right]
$$

of weight $k$ is denoted by $\left[g_{i_{1}}, g_{i_{2}}, \ldots, g_{i_{k}}\right]$.

- For any $\mathbb{Z}$-module $M$ and a commutative ring $R$, we denote $M \otimes_{\mathbb{Z}} R$ by the symbol obtained by attaching a subscript $R$ to $M$, like $M_{R}$ or $M^{R}$. Similarly, for any $\mathbb{Z}$-linear map $f: A \rightarrow B$, the induced $R$-linear map $A_{R} \rightarrow B_{R}$ is denoted by $f_{R}$ or $f^{R}$. 
- For a semisimple $G$-module $M$ and an irreducible $G$-module $N$, we denote by $[M: N]$ the multiplicity of $N$ in the irreducible decomposition of $M$.

\section{Johnson homomorphisms of the mapping class groups and the automorphism group of free groups}

The first aim of this section is to recall the notion of Johnson homomorphisms for the mapping class groups of surfaces and the automorphism groups of free groups. The second one is to review the second author's results on the structure of the Johnson cokernels with respect to the lower central series of the IA-automorphism group of free groups. Third, we obtain a diagram (2) at the end of this section by using Hain's result (Theorem 3.5). Through this diagram, we can compare the structure of the Johnson cokernels of the mapping class groups of surfaces with those for the automorphism groups of free groups. In Section 7.1, we give a new class in the Johnson cokernels for the mapping class groups of surfaces by using this diagram.

\subsection{Mapping class groups of surfaces}

Here we recall some properties of the mapping class groups of surfaces. For any integer $g \geq 1$, let $\Sigma_{g, 1}$ be the compact oriented surface of genus $g$ with one boundary component. We denote by $\mathcal{M}_{g, 1}$ the mapping class group of $\Sigma_{g, 1}$. Namely, $\mathcal{M}_{g, 1}$ is the group of isotopy classes of orientation-preserving diffeomorphisms of $\Sigma_{g, 1}$ that fix the boundary pointwise.

The mapping class group $\mathcal{M}_{g, 1}$ has an important normal subgroup called the Torelli group. Let $\mu_{\mathcal{M}}: \mathcal{M}_{g, 1} \rightarrow \operatorname{Aut}\left(H_{1}\left(\Sigma_{g, 1}, \mathbb{Z}\right)\right)$ be the classical representation of $\mathcal{M}_{g, 1}$ induced from the action of $\mathcal{M}_{g, 1}$ on the integral first homology group $H_{1}\left(\Sigma_{g, 1}, \mathbb{Z}\right)$ of $\Sigma_{g, 1}$. The kernel of $\mu_{\mathcal{M}}$ is called the Torelli group, denoted by $\mathcal{I}_{g, 1}$. Namely, $\mathcal{I}_{g, 1}$ consists of mapping classes of $\Sigma_{g, 1}$ that act on $H_{1}\left(\Sigma_{g, 1}, \mathbb{Z}\right)$ trivially.

Let us observe the image of $\mu_{\mathcal{M}}$. Take a base point $*$ of $\Sigma_{g, 1}$ on the boundary. Then the fundamental group $\pi_{1}\left(\Sigma_{g, 1}, *\right)$ of $\Sigma_{g, 1}$ is a free group of rank $2 g$. We fix a basis $x_{1}, \ldots, x_{2 g}$ of $\pi_{1}\left(\Sigma_{g, 1}, *\right)$ as shown Figure 1 .

Then the homology classes $e_{1}, \ldots, e_{2 g}$ of $x_{1}, \ldots, x_{2 g}$ form a symplectic basis of the homology group $H_{1}\left(\Sigma_{g, 1}, \mathbb{Z}\right)$. Using this symplectic basis, we can identify $\operatorname{Aut}\left(H_{1}\left(\Sigma_{g, 1}, \mathbb{Z}\right)\right)$ as the general linear group $\mathrm{GL}(2 g, \mathbb{Z})$. Under this identification, the image of $\mu_{M}$ is considered as the symplectic group

$$
\operatorname{Sp}(2 g, \mathbb{Z}):=\left\{X \in \mathrm{GL}(2 g, \mathbb{Z}) \mid{ }^{t} X J X=J\right\} \quad \text { for } J=\left(\begin{array}{cc}
0 & I_{g} \\
-I_{g} & 0
\end{array}\right),
$$




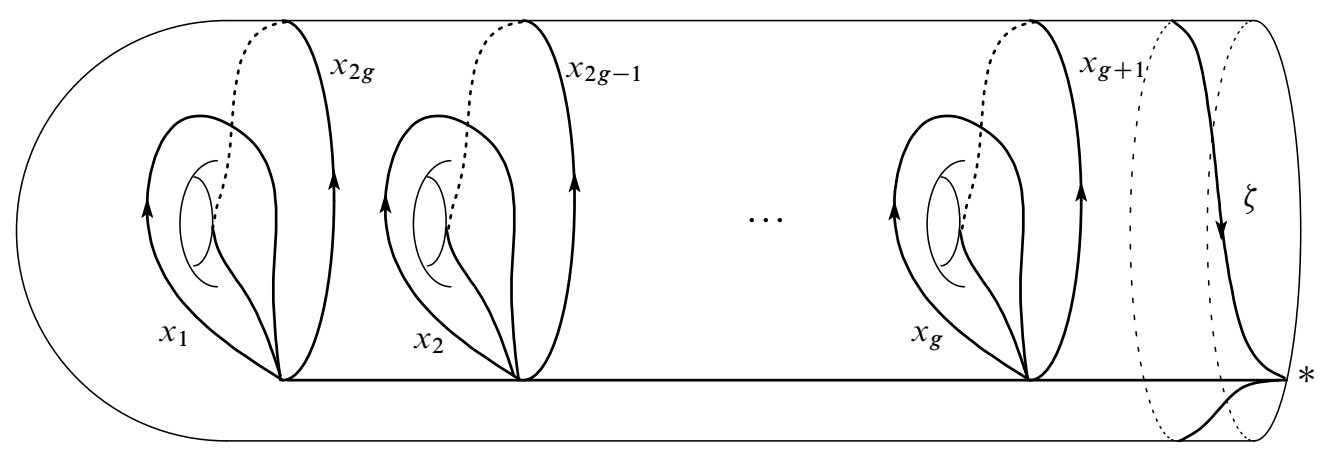

Figure 1: Generators $x_{1}, \ldots, x_{2 g}$ of $\pi_{1}\left(\Sigma_{g, 1}, *\right)$ and a simple closed curve $\zeta$

where $I_{g}$ is the identity matrix of degree $g$.

Next, we consider an embedding of the mapping class group $\mathcal{M}_{g, 1}$ into the automorphism group of a free group of rank $2 g$. For $n \geq 2$ let $F_{n}$ be a free group of rank $n$ with basis $x_{1}, \ldots, x_{n}$. We denote by $\operatorname{Aut}\left(F_{n}\right)$ the automorphism group of $F_{n}$. Let $H$ be the abelianization $H_{1}\left(F_{n}, \mathbb{Z}\right)$ of $F_{n}$ and $\mu: \operatorname{Aut}\left(F_{n}\right) \rightarrow \operatorname{Aut}(H)$ a natural homomorphism induced from the abelianization map $F_{n} \rightarrow H$. Throughout the paper, we identify $\operatorname{Aut}(H)$ with the general linear group $\operatorname{GL}(n, \mathbb{Z})$ by fixing a basis $e_{1}, \ldots, e_{n}$ of $H$ induced from the basis $x_{1}, \ldots, x_{n}$ of $F_{n}$. By a classical work of Nielsen [25], a finite presentation of $\operatorname{Aut}\left(F_{n}\right)$ is obtained. Observing the images of the generators of Nielsen's presentation, we see that $\rho$ is surjective. The kernel $\mathrm{IA}_{n}$ of $\rho$ is called the IA-automorphism group of $F_{n}$. The IA-automorphism group $\mathrm{IA}_{n}$ is a free group analogue of the Torelli group $\mathcal{I}_{g, 1}$.

Now, throughout the paper, we identify $\pi_{1}\left(\Sigma_{g, 1}, *\right)$ with $F_{2 g}$, and $H_{1}\left(\Sigma_{g, 1}, \mathbb{Z}\right)$ with $H$ for $n=2 g$ using the basis above. Then the action of $\mathcal{M}_{g, 1}$ on $\pi_{1}\left(\Sigma_{g, 1}, *\right)=F_{2 g}$ induces a natural homomorphism

$$
\varphi: \mathcal{M}_{g, 1} \rightarrow \operatorname{Aut}\left(F_{2 g}\right) .
$$

By a classical work due to Dehn and Nielsen, it is known that $\varphi$ is injective. More precisely, we have:

Theorem 3.1 (Dehn and Nielsen) For any $g \geq 1$, we have

$$
\varphi\left(\mathcal{M}_{g, 1}\right)=\left\{\sigma \in \operatorname{Aut}\left(F_{2 g}\right) \mid \zeta^{\sigma}=\zeta\right\}
$$

where $\zeta=\left[x_{1}, x_{2 g}\right]\left[x_{2}, x_{2 g-1}\right] \cdots\left[x_{g}, x_{g+1}\right] \in F_{2 g}$, namely $\zeta$ is a homotopy class of a simple closed curve on $\Sigma_{g, 1}$ parallel to the boundary. 
For $n=2 g$, we have $\mu_{\mathcal{M}}=\mu \circ \varphi: \mathcal{M}_{g, 1} \rightarrow \operatorname{Sp}(2 g, \mathbb{Z})$, and a commutative diagram:

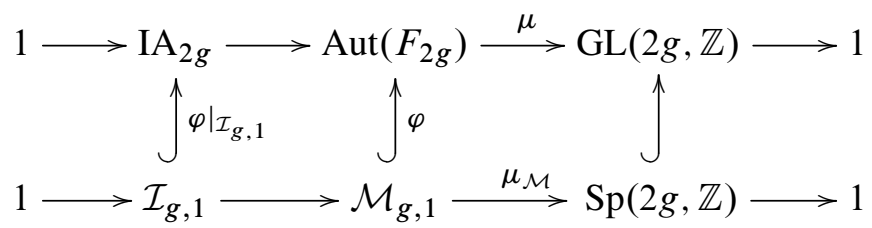

\subsection{Free Lie algebras}

In this subsection, we recall the free Lie algebra generated by $H$, and its derivation algebra. (See Serre [29] and Reutenauer [27] for basic material concerning the free Lie algebra for instance.)

Let $\Gamma_{n}(1) \supset \Gamma_{n}(2) \supset \cdots$ be the lower central series of a free group $F_{n}$ defined by the rule

$$
\Gamma_{n}(1):=F_{n}, \quad \Gamma_{n}(k):=\left[\Gamma_{n}(k-1), F_{n}\right], \quad k \geq 2 .
$$

We denote by $\mathcal{L}_{n}(k):=\Gamma_{n}(k) / \Gamma_{n}(k+1)$ the $k^{\text {th }}$ graded quotient of the lower central series of $F_{n}$, and by $\mathcal{L}_{n}:=\bigoplus_{k \geq 1} \mathcal{L}_{n}(k)$ the associated graded sum. The degree 1 part $\mathcal{L}_{n}(1)$ of $\mathcal{L}_{n}$ is just $H$. Classically, Magnus showed that each of $\mathcal{L}_{n}(k)$ is a free abelian, and Witt [30] gave its rank as follows.

$$
\operatorname{rank}_{\mathbb{Z}}\left(\mathcal{L}_{n}(k)\right)=\frac{1}{k} \sum_{d \mid k} \operatorname{Möb}(d) n^{\frac{k}{d}},
$$

where Möb is the Möbius function. For any $k, l \geq 1$, let us consider a bilinear alternating map

$$
[\cdot, \cdot]_{\text {Lie }}: \mathcal{L}_{n}(k) \times \mathcal{L}_{n}(l) \rightarrow \mathcal{L}_{n}(k+l)
$$

defined by $[[\alpha],[\beta]]_{\text {Lie }}:=[[\alpha, \beta]]$ for any $[\alpha] \in \mathcal{L}_{n}(k)$ and $[\beta] \in \mathcal{L}_{n}(l)$, where $[\alpha, \beta]$ is a commutator in $F_{n}$, and $[[\alpha, \beta]]$ is a coset class of $[\alpha, \beta]$ in $\mathcal{L}_{n}(k+l)$. Then $[\cdot, \cdot]_{\text {Lie }}$ induces a graded Lie algebra structure of the graded sum $\mathcal{L}_{n}$. By a classical work of Magnus, the Lie algebra $\mathcal{L}_{n}$ is isomorphic to the free Lie algebra generated by $H$.

The Lie algebra $\mathcal{L}_{n}$ is considered as a Lie subalgebra of the tensor algebra generated by $H$ as follows. Let $T(H):=\mathbb{Z} \oplus H \oplus H^{\otimes 2} \oplus \cdots$ be the tensor algebra of $H$ over $\mathbb{Z}$. Then $T(H)$ is the universal enveloping algebra of the free Lie algebra $\mathcal{L}_{n}$, and the natural map $\iota: \mathcal{L}_{n} \rightarrow T(H)$ defined by $[X, Y] \mapsto X \otimes Y-Y \otimes X$ for $X, Y \in \mathcal{L}_{n}$ is an injective graded Lie algebra homomorphism. We denote by $\iota_{k}$ the homomorphism of degree $k$ part of $\iota$, and consider $\mathcal{L}_{n}(k)$ as a submodule $H^{\otimes k}$ through $\iota_{k}$. 
Here, we recall the derivation algebra of the free Lie algebra. Let $\operatorname{Der}\left(\mathcal{L}_{n}\right)$ be the graded Lie algebra of derivations of $\mathcal{L}_{n}$. Namely,

$$
\operatorname{Der}\left(\mathcal{L}_{n}\right):=\left\{f: \mathcal{L}_{n} \stackrel{\mathbb{Z} \text {-linear }}{\longrightarrow} \mathcal{L}_{n} \mid f([a, b])=[f(a), b]+[a, f(b)], a, b \in \mathcal{L}_{n}\right\} .
$$

For $k \geq 0$, the degree $k$ part of $\operatorname{Der}\left(\mathcal{L}_{n}\right)$ is defined to be

$$
\operatorname{Der}\left(\mathcal{L}_{n}\right)(k):=\left\{f \in \operatorname{Der}\left(\mathcal{L}_{n}\right) \mid f(a) \in \mathcal{L}_{n}(k+1), a \in H\right\} .
$$

Then, we have

$$
\operatorname{Der}\left(\mathcal{L}_{n}\right)=\bigoplus_{k \geq 0} \operatorname{Der}\left(\mathcal{L}_{n}\right)(k)
$$

and can consider $\operatorname{Der}\left(\mathcal{L}_{n}\right)(k)$ as $\operatorname{Hom}_{\mathbb{Z}}\left(H, \mathcal{L}_{n}(k+1)\right)=H^{*} \otimes_{\mathbb{Z}} \mathcal{L}_{n}(k+1)$ for each $k \geq 1$ by the universality of the free Lie algebra. Let $\operatorname{Der}^{+}\left(\mathcal{L}_{n}\right)$ be a graded Lie subalgebra of $\operatorname{Der}\left(\mathcal{L}_{n}\right)(k)$ with positive degree. (See Bourbaki [3, Chapter II, Section 8].)

\section{3 (Higher) Johnson homomorphisms}

First we recall the Johnson filtration and the Johnson homomorphisms of the automorphism group of a free group. Then we consider those of the mapping class group.

For each $k \geq 1$, let $N_{n, k}:=F_{n} / \Gamma_{n}(k+1)$ of $F_{n}$ be the free nilpotent group of class $k$ and rank $n$, and $\operatorname{Aut}\left(N_{n, k}\right)$ its automorphism group. Since the subgroup $\Gamma_{n}(k+1)$ is characteristic in $F_{n}$, the group $\operatorname{Aut}\left(F_{n}\right)$ naturally acts on $N_{n, k}$ from the right. This action induces a homomorphism $\operatorname{Aut}\left(F_{n}\right) \rightarrow \operatorname{Aut}\left(N_{n, k}\right)$. Let $\mathcal{A}_{n}(k)$ be the kernel of this homomorphism. Then the groups $\mathcal{A}_{n}(k)$ define a descending filtration

$$
\mathrm{IA}_{n}=\mathcal{A}_{n}(1) \supset \mathcal{A}_{n}(2) \supset \cdots .
$$

This filtration is called the Johnson filtration of $\operatorname{Aut}\left(F_{n}\right)$. Set

$$
\operatorname{gr}^{k}\left(\mathcal{A}_{n}\right):=\mathcal{A}_{n}(k) / \mathcal{A}_{n}(k+1) .
$$

Andreadakis [1] originally studied the Johnson filtration, and obtained basic and important properties of it as follows:

Theorem 3.2 (Andreadakis [1])

(i) For any $k, l \geq 1, \sigma \in \mathcal{A}_{n}(k)$ and $x \in \Gamma_{n}(l), x^{-1} x^{\sigma} \in \Gamma_{n}(k+l)$.

(ii) For any $k, l \geq 1,\left[\mathcal{A}_{n}(k), \mathcal{A}_{n}(l)\right] \subset \mathcal{A}_{n}(k+l)$. In other words, the Johnson filtration is a descending central filtration of $\mathrm{IA}_{n}$.

(iii) For any $k \geq 1, \operatorname{gr}^{k}\left(\mathcal{A}_{n}\right)$ is a free abelian group of finite rank. 
In order to study the structure of $\operatorname{gr}^{k}\left(\mathcal{A}_{n}\right)$, the $k^{\text {th }}$ Johnson homomorphism of $\operatorname{Aut}\left(F_{n}\right)$ is defined as follows.

Definition 3.3 For each $k \geq 1$, define a homomorphism

$$
\begin{aligned}
\tilde{\tau}_{k}: \mathcal{A}_{n}(k) & \rightarrow \operatorname{Hom}_{\mathbb{Z}}\left(H, \mathcal{L}_{n}(k+1)\right), \\
\sigma & \mapsto\left(x \bmod \Gamma_{n}(2) \mapsto x^{-1} x^{\sigma} \bmod \Gamma_{n}(k+2)\right), \quad x \in F_{n} .
\end{aligned}
$$

Then the kernel of $\tilde{\tau}_{k}$ is just $\mathcal{A}_{n}(k+1)$. Hence it induces an injective homomorphism

$$
\tau_{k}: \operatorname{gr}^{k}\left(\mathcal{A}_{n}\right) \hookrightarrow \operatorname{Hom}_{\mathbb{Z}}\left(H, \mathcal{L}_{n}(k+1)\right)=H^{*} \otimes_{\mathbb{Z}} \mathcal{L}_{n}(k+1) .
$$

This homomorphism is called the $k^{\text {th }}$ Johnson homomorphism of $\operatorname{Aut}\left(F_{n}\right)$.

Here we consider actions of $\operatorname{GL}(n, \mathbb{Z})=\operatorname{Aut}\left(F_{n}\right) / \mathrm{IA}_{n}$. First, since each term of the lower central series of $F_{n}$ is a characteristic subgroup, $\operatorname{Aut}\left(F_{n}\right)$ naturally acts on it, and hence on each of the graded quotients $\mathcal{L}_{n}(k)$. By (i) of Theorem 3.2, we see that the action of $\mathrm{IA}_{n}$ on $\mathcal{L}_{n}(k)$ is trivial. Thus the action of $\operatorname{GL}(n, \mathbb{Z})=\operatorname{Aut}\left(F_{n}\right) / \mathrm{IA}_{n}$ on $\mathcal{L}_{n}(k)$ is well-defined.

On the other hand, since each term of the Johnson filtration is a normal subgroup of $\operatorname{Aut}\left(F_{n}\right)$, the group $\operatorname{Aut}\left(F_{n}\right)$ naturally acts on $\mathcal{A}_{n}(k)$ by conjugation, and hence each of the graded quotient $\mathrm{gr}^{k}\left(\mathcal{A}_{n}\right)$. By (ii) of Theorem 3.2, we see that the action of $\mathrm{IA}_{n}$ on $\operatorname{gr}^{k}\left(\mathcal{A}_{n}\right)$ is trivial. Hence, the quotient group $\operatorname{GL}(n, \mathbb{Z})=\operatorname{Aut}\left(F_{n}\right) / \mathrm{IA}_{n}$ naturally acts on each $\operatorname{gr}^{k}\left(\mathcal{A}_{n}\right)$. With respect to the actions above, we see that the Johnson homomorphism $\tau_{k}$ is $\operatorname{GL}(n, \mathbb{Z})$-equivariant for each $k \geq 1$.

Furthermore, we remark that the sum of the Johnson homomorphisms forms a Lie algebra homomorphism as follows. Let $\operatorname{gr}\left(\mathcal{A}_{n}\right):=\bigoplus_{k \geq 1} \operatorname{gr}^{k}\left(\mathcal{A}_{n}\right)$ be the graded sum of $\operatorname{gr}^{k}\left(\mathcal{A}_{n}\right)$. The graded sum $\operatorname{gr}\left(\mathcal{A}_{n}\right)$ has a graded Lie algebra structure induced from the commutator bracket on $\mathrm{IA}_{n}$ by an argument similar to that of the free Lie algebra $\mathcal{L}_{n}$. Then the sum of the Johnson homomorphisms

$$
\tau:=\bigoplus_{k \geq 1} \tau_{k}: \operatorname{gr}\left(\mathcal{A}_{n}\right) \rightarrow \operatorname{Der}^{+}\left(\mathcal{L}_{n}\right)
$$

is a graded Lie algebra homomorphism. (See also [22, Theorem 4.8].)

In the following, we consider three central subfiltrations of the Johnson filtration of $\operatorname{Aut}\left(F_{n}\right)$, and "restrictions" of the Johnson homomorphism $\tau_{k}$.

The first one is the lower central series of $\mathrm{IA}_{n}$. Let $\mathcal{A}_{n}^{\prime}(k)$ be the lower central series of $\mathrm{IA}_{n}$ with $\mathcal{A}_{n}^{\prime}(1)=\mathrm{IA}_{n}$. Since the Johnson filtration is central, $\mathcal{A}_{n}^{\prime}(k) \subset \mathcal{A}_{n}(k)$ for each $k \geq 1$. Set $\operatorname{gr}^{k}\left(\mathcal{A}_{n}^{\prime}\right):=\mathcal{A}_{n}^{\prime}(k) / \mathcal{A}_{n}^{\prime}(k+1)$. Then $\operatorname{GL}(n, \mathbb{Z})$ naturally acts on 
each of $\operatorname{gr}^{k}\left(\mathcal{A}_{n}^{\prime}\right)$, and the restriction of $\tilde{\tau}_{k}$ to $\mathcal{A}_{n}^{\prime}(k)$ induces a $\operatorname{GL}(n, \mathbb{Z})$-equivariant homomorphism

$$
\tau_{k}^{\prime}: \operatorname{gr}^{k}\left(\mathcal{A}_{n}^{\prime}\right) \rightarrow H^{*} \otimes_{\mathbb{Z}} \mathcal{L}_{n}(k+1) .
$$

We also call $\tau_{k}^{\prime}$ the Johnson homomorphism of $\operatorname{Aut}\left(F_{n}\right)$. Let $i_{k}: \operatorname{gr}^{k}\left(\mathcal{A}_{n}^{\prime}\right) \rightarrow \operatorname{gr}^{k}\left(\mathcal{A}_{n}\right)$ be the homomorphism induced from the inclusion $\mathcal{A}_{n}^{\prime}(k) \hookrightarrow \mathcal{A}_{n}(k)$. Then $\tau_{k}^{\prime}=\tau_{k} \circ i_{k}$ for each $k \geq 1$. Similarly to the sum $\tau$ of the $\tau_{k}$, the sum $\tau^{\prime}:=\bigoplus_{k \geq 1} \tau_{k}^{\prime}: \operatorname{gr}\left(\mathcal{A}_{n}^{\prime}\right) \rightarrow$ $\operatorname{Der}^{+}\left(\mathcal{L}_{n}\right)$ is a graded Lie algebra homomorphism.

Let $\mathcal{C}_{n}(k)$ be a quotient module of $H^{\otimes k}$ by the action of cyclic group $\mathrm{Cyc}_{k}$ of order $k$ on the components:

$$
\mathcal{C}_{n}(k)=H^{\otimes k} /\left\langle a_{1} \otimes a_{2} \otimes \cdots \otimes a_{k}-a_{2} \otimes a_{3} \otimes \cdots \otimes a_{k} \otimes a_{1} \mid a_{i} \in H\right\rangle
$$

In [28], we determined the cokernel of the rational Johnson homomorphisms $\tau_{k}^{\prime}$ in stable range. Namely, we have:

Theorem 3.4 (Satoh [28]) For any $k \geq 2$ and $n \geq k+2, \operatorname{Coker}\left(\tau_{k, \mathbb{Q}}^{\prime}\right) \cong \mathcal{C}_{n}^{\mathbb{Q}}(k)$.

We also remark that in our previous paper [5], we studied the GL-irreducible decomposition of $\mathcal{C}_{n}^{\mathbb{Q}}(k)$. For more details, see Lemma 7.2 and Proposition 7.3.

Next, we consider the Johnson filtration of the mapping class group. By Dehn and Nielsen's classical work, we can consider $\mathcal{M}_{g, 1}$ as a subgroup of $\operatorname{Aut}\left(F_{2 g}\right)$ as above. Under this embedding, set $\mathcal{M}_{g, 1}(k):=\mathcal{M}_{g, 1} \cap \mathcal{A}_{2 g}(k)$ for each $k \geq 1$. Then we have a descending filtration

$$
\mathcal{I}_{g, 1}=\mathcal{M}_{g, 1}(1) \supset \mathcal{M}_{g, 1}(2) \supset \cdots
$$

of the Torelli group $\mathcal{I}_{g, 1}$. This filtration is called the Johnson filtration of $\mathcal{M}_{g, 1}$. Set $\operatorname{gr}^{k}\left(\mathcal{M}_{g, 1}\right):=\mathcal{M}_{g, 1}(k) / \mathcal{M}_{g, 1}(k+1)$. For each $k \geq 1$, the mapping class group $\mathcal{M}_{g, 1}$ acts on $\operatorname{gr}^{k}\left(\mathcal{M}_{g, 1}\right)$ by conjugation. This action induces that of $\operatorname{Sp}(2 g, \mathbb{Z})=\mathcal{M}_{g, 1} / \mathcal{I}_{g, 1}$ on it.

By an argument similar to that for $\operatorname{Aut}\left(F_{n}\right)$, the Johnson homomorphisms of $\mathcal{M}_{g, 1}$ are defined as follows. For $n=2 g$ and $k \geq 1$, consider the restriction of $\tilde{\tau}_{k}: \mathcal{A}_{2 g}(k) \rightarrow$ $\operatorname{Hom}_{\mathbb{Z}}\left(H, \mathcal{L}_{2 g}(k+1)\right)$ to $\mathcal{M}_{g, 1}(k)$. Then its kernel is just $\mathcal{M}_{g, 1}(k+1)$. Hence we obtain an injective homomorphism

$$
\tau_{k}^{\mathcal{M}}: \mathrm{gr}^{k}\left(\mathcal{M}_{g, 1}\right) \hookrightarrow \operatorname{Hom}_{\mathbb{Z}}\left(H, \mathcal{L}_{2 g}(k+1)\right)=H^{*} \otimes_{\mathbb{Z}} \mathcal{L}_{2 g}(k+1) .
$$

The homomorphism $\tau_{k}^{\mathcal{M}}$ is $\operatorname{Sp}(2 g, \mathbb{Z})$-equivariant, and is called the $k^{\text {th }}$ Johnson homomorphism of $\mathcal{M}_{g, 1}$. If we consider a $\operatorname{GL}(2 g, \mathbb{Z})$-module $H$ as a $\operatorname{Sp}(2 g, \mathbb{Z})$ module, then $H^{*} \cong H$ by the Poincaré duality. Hence, in the following, we canonically identify the target $H^{*} \otimes_{\mathbb{Z}} \mathcal{L}_{2 g}(k+1)$ of $\tau_{k}^{\mathcal{M}}$ with $H \otimes_{\mathbb{Z}} \mathcal{L}_{2 g}(k+1)$. 
Historically, the Johnson filtration of $\operatorname{Aut}\left(F_{n}\right)$ was originally studied by Andreadakis [1] in the 1960s as mentioned above. On the other hand, study of the Johnson filtration and the Johnson homomorphisms of $\mathcal{M}_{g, 1}$ was begun in the 1980 s by D Johnson [11], who determined the abelianization of the Torelli subgroup of the mapping class group of a surface in [14]. In particular, he showed that $\operatorname{Im}\left(\tau_{1}^{\mathcal{M}}\right) \cong \Lambda^{3} H$ as an $\operatorname{Sp}(2 g, \mathbb{Z})$-module, and this gives the free part of $H_{1}\left(\mathcal{I}_{g, 1}, \mathbb{Z}\right)$.

Now, let us recall the fact that the image of $\tau_{k}^{\mathcal{M}}$ is contained in a certain $\operatorname{Sp}(2 g, \mathbb{Z})-$ submodule of $H \otimes_{\mathbb{Z}} \mathcal{L}_{2 g}(k+1)$, due to Morita [22]. In general, for any $n \geq 1$, let $H \otimes_{\mathbb{Z}} \mathcal{L}_{n}(k+1) \rightarrow \mathcal{L}_{n}(k+2)$ be a $\operatorname{GL}(n, \mathbb{Z})$-equivariant homomorphism defined by

$$
a \otimes X \mapsto[a, X], \quad \text { for } a \in H, X \in \mathcal{L}_{n}(k+1) .
$$

For $n=2 g$, we denote by $\mathfrak{h}_{g, 1}(k)$ the kernel of this homomorphism:

$$
\mathfrak{h}_{g, 1}(k):=\operatorname{Ker}\left(H \otimes_{\mathbb{Z}} \mathcal{L}_{2 g}(k+1) \rightarrow \mathcal{L}_{2 g}(k+2)\right)
$$

Then Morita [22] showed that the image $\operatorname{Im}\left(\tau_{k}^{\mathcal{M}}\right)$ is contained in $\mathfrak{h}_{g, 1}(k)$. Therefore, to determine how different $\operatorname{Im}\left(\tau_{k}^{\mathcal{M}}\right)$ is from $\mathfrak{h}_{g, 1}(k)$ is one of the most basic problems. Throughout the paper, the cokernel $\operatorname{Coker}\left(\tau_{k}^{\mathcal{M}}\right)$ of $\tau_{k}^{\mathcal{M}}$ always means the quotient $\operatorname{Sp}(2 g, \mathbb{Z})$-module $\mathfrak{h}_{g, 1}(k) / \operatorname{Im}\left(\tau_{k}^{\mathcal{M}}\right)$. So far, the $\operatorname{Sp}-\operatorname{module}$ structure of $\operatorname{Coker}\left(\tau_{k, \mathbb{Q}}^{\mathcal{M}}\right)$ is determined for $1 \leq k \leq 4$ as follows.

\begin{tabular}{l|l|l|l}
$k$ & $\operatorname{Im}\left(\tau_{k, \mathbb{Q}}^{\mathcal{M}}\right)$ & $\operatorname{Coker}\left(\tau_{k, \mathbb{Q}}^{\mathcal{M}}\right)$ & \\
\hline 1 & {$\left[1^{3}\right] \oplus[1]$} & 0 & Johnson [11] \\
2 & {$\left[2^{2}\right] \oplus\left[1^{2}\right] \oplus[0]$} & 0 & Morita [21], Hain [8] \\
3 & {$\left[3,1^{2}\right] \oplus[2,1]$} & {$[3]$} & Asada and Nakamura[2], \\
4 & {$[4,2] \oplus\left[3,1^{3}\right] \oplus\left[2^{3}\right] \oplus 2[3,1] \oplus\left[2,1^{2}\right] \oplus 2[2]$} & {$\left[2,1^{2}\right] \oplus[2]$} & Hain [8] \\
Morita [23]
\end{tabular}

Morita [22] showed that the symmetric tensor product $S^{k} H_{\mathbb{Q}}$ appears in the $\mathrm{Sp}-$ irreducible decomposition of $\operatorname{Coker}\left(\tau_{k, \mathbb{Q}}^{\mathcal{M}}\right)$ for odd $k \geq 3$ using the Morita trace map. In general, however, to determine the cokernel of $\tau_{k}^{\mathcal{M}}$ is a difficult problem.

Here, we recall a remarkable result of Hain. As an $\operatorname{Sp}(2 g, \mathbb{Z})$-module, we consider $\mathfrak{h}_{g, 1}(k)$ as a submodule of the degree $k$ part $\operatorname{Der}\left(\mathcal{L}_{n}\right)(k)$ of the derivation algebra of $\mathcal{L}_{n}$. On the other hand, the graded sum $\mathfrak{h}_{g, 1}:=\bigoplus_{k \geq 1} \mathfrak{h}_{g, 1}(k)$ naturally has a Lie subalgebra structure of $\operatorname{Der}^{+}\left(\mathcal{L}_{n}\right)$. Therefore we obtain a graded Lie algebra homomorphism

$$
\tau^{\mathcal{M}}:=\bigoplus_{k \geq 1} \tau_{k}^{\mathcal{M}}: \operatorname{gr}\left(\mathcal{M}_{g, 1}\right) \rightarrow \mathfrak{h}_{g, 1}
$$


Then we have:

Theorem 3.5 (Hain [8]) The Lie subalgebra $\operatorname{Im}\left(\tau_{\mathbb{Q}}^{\mathcal{M}}\right)$ is generated by the degree one part $\operatorname{Im}\left(\tau_{1, \mathbb{Q}}^{\mathcal{M}}\right)=\Lambda^{3} H_{\mathbb{Q}}$ as a Lie algebra.

Finally, we consider the lower central series of the Torelli group, and reformulate Hain's result above. Let $\mathcal{M}_{g, 1}^{\prime}(k)$ be the lower central series of $\mathcal{I}_{g, 1}$, and set

$$
\operatorname{gr}^{k}\left(\mathcal{M}_{g, 1}^{\prime}\right):=\mathcal{M}_{g, 1}^{\prime}(k) / \mathcal{M}_{g, 1}^{\prime}(k+1)
$$

for $k \geq 1$. Let $\tau_{k}^{\prime \mathcal{M}}: \operatorname{gr}^{k}\left(\mathcal{M}_{g, 1}^{\prime}\right) \rightarrow H \otimes_{\mathbb{Z}} \mathcal{L}_{2 g}(k+1)$ be the Sp-equivariant homomorphism induced from the restriction of $\tilde{\tau}_{k}$ to $\mathcal{M}_{g, 1}^{\prime}(k)$. Then we have:

Proposition 3.6 (Hain [8]) We have $\operatorname{Im}\left(\tau_{k, \mathbb{Q}}^{\mathcal{M}}\right)=\operatorname{Im}\left(\tau_{k, \mathbb{Q}}^{\prime \mathcal{M}}\right)$ for each $k \geq 1$.

For $n=2 g$, we have the following commutative diagram:

$$
\operatorname{Im} \tau_{k, \mathbb{Q}}^{\prime} \longleftrightarrow H_{\mathbb{Q}}^{*} \otimes_{\mathbb{Q}} \mathcal{L}_{2 g}^{\mathbb{Q}}(k+1) \rightarrow H_{\mathbb{Q}}^{\otimes k} \longrightarrow \mathcal{C}_{2 g}^{\mathbb{Q}}(k)
$$

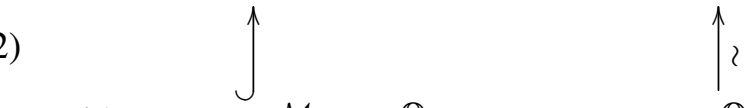

$$
\operatorname{Im} \tau_{k, \mathbb{Q}}^{\mathcal{M}}=\operatorname{Im} \tau_{k, \mathbb{Q}}^{\prime \mathcal{M}} \hookrightarrow \mathfrak{h}_{g, 1}^{\mathbb{Q}}(k) \hookrightarrow H_{\mathbb{Q}} \otimes \mathbb{Q} \mathcal{L}_{2 g}^{\mathbb{Q}}(k+1) \longrightarrow \mathcal{L}_{2 g}^{\mathbb{Q}}(k+1)
$$

\section{Highest weight theory for $\operatorname{Sp}(2 g, \mathbb{Q})$}

In order to detect a series of Sp-irreducible representations in the Johnson cokernels for the mapping class groups of surfaces, we use some representation theory of the symplectic group, for example, the Brauer-Schur-Weyl duality. In this section, first, we review the highest weight theory for $\operatorname{Sp}(2 g, \mathbb{Q})$, a classification of isomorphism classes of Sp-irreducible (rational) representations and the branching rules for GL to $\mathrm{Sp}$. These branching rules are used to calculate the multiplicities of Sp-irreducible representations in $\mathfrak{h}_{g, 1}^{\mathbb{Q}}$ in Section 7.2. Second, we review the classical Schur-Weyl duality for GL and the symmetric group, the Brauer-Schur-Weyl duality for Sp, and the Brauer algebra. From these results, we can obtain a description of a generating set of the space of Sp-maximal vectors in $H_{\mathbb{Q}}^{\otimes k}$. This fact is used in Section 7.3 when we detect a series of $\mathrm{Sp}$-irreducible representations in the Johnson cokernels. Third, in the last of this section, we explain a result for the irreducible characters of the Brauer algebras. This enables us to calculate the multiplicities of the Sp-irreducible representations in $H_{\mathbb{Q}}^{\otimes k}$. In particular, we obtain the dimension of Sp-invariant part of $H_{\mathbb{Q}}^{\otimes k}$, which is another proof of Morita's result [23, Lemma 4.1]. 


\subsection{Irreducible highest weight modules for $\operatorname{Sp}(2 g, \mathbb{Q})$}

Let us consider the general linear group $\operatorname{GL}(n, \mathbb{Q})$ and the symplectic group

$$
\operatorname{Sp}(2 g, \mathbb{Q}):=\left\{X \in \mathrm{GL}(2 g, \mathbb{Q}) \mid{ }^{t} X J X=J\right\} \quad \text { for } J=\left(\begin{array}{cc}
0 & I_{g} \\
-I_{g} & 0
\end{array}\right),
$$

where $I_{g}$ is the identity matrix of degree $g$. We fix a maximal torus

$$
T_{n}=\left\{\operatorname{diag}\left(x_{1}, \ldots, x_{n}\right) \mid x_{j} \neq 0,1 \leq j \leq n\right\}
$$

of $\operatorname{GL}(n, \mathbb{Q})$. The intersection $\operatorname{Sp}(2 g, \mathbb{Q}) \cap T_{2 g}=\left\{\operatorname{diag}\left(x_{1}, \ldots, x_{n}, x_{n}^{-1}, \ldots, x_{1}^{-1}\right)\right\}$ gives a maximal torus of $\operatorname{Sp}(2 g, \mathbb{Q})$. We also fix this maximal torus and write $T_{2 g}^{\mathrm{Sp}}$.

We define one-dimensional representations $\varepsilon_{i}$ of $T_{n}$ by $\varepsilon_{i}\left(\operatorname{diag}\left(x_{1}, \ldots, x_{n}\right)\right)=x_{i}$. Then

$$
\begin{aligned}
& P_{\mathrm{GL}(n, \mathbb{Q})}:=\left\{\lambda_{1} \varepsilon_{1}+\cdots+\lambda_{n} \varepsilon_{n} \mid \lambda_{i} \in \mathbb{Z}, 1 \leq i \leq n\right\} \cong \mathbb{Z}^{n}, \\
& P_{\mathrm{GL}(n, \mathbb{Q})}^{+}:=\left\{\lambda_{1} \varepsilon_{1}+\cdots+\lambda_{n} \varepsilon_{n} \in P_{\mathrm{GL}(n, \mathbb{Q})} \mid \lambda_{1} \geq \lambda_{2} \geq \cdots \geq \lambda_{n}\right\}
\end{aligned}
$$

give the weight lattice and the set of dominant integral weights of $\operatorname{GL}(n, \mathbb{Q})$, respectively. If $n=2 g$, we can restrict $\varepsilon_{i}$ to $T_{2 g}^{\mathrm{Sp}}$ for $1 \leq i \leq g$. Then

$$
\begin{aligned}
& P_{\mathrm{Sp}(2 g, \mathbb{Q})}:=\left\{\lambda_{1} \varepsilon_{1}+\cdots+\lambda_{g} \varepsilon_{g} \mid \lambda_{i} \in \mathbb{Z}, 1 \leq i \leq g\right\} \cong \mathbb{Z}^{g}, \\
& P_{\mathrm{Sp}(2 g, \mathbb{Q})}^{+}:=\left\{\lambda_{1} \varepsilon_{1}+\cdots+\lambda_{g} \varepsilon_{g} \in P_{\mathrm{Sp}(2 g, \mathbb{Q})} \mid \lambda_{1} \geq \lambda_{2} \geq \cdots \geq \lambda_{g} \geq 0\right\}
\end{aligned}
$$

give the weight lattice and the set of dominant integral weights of $\operatorname{Sp}(2 g, \mathbb{Q})$ respectively. In particular, there exists a bijection between $P_{\mathrm{Sp}(2 g, \mathbb{Q})}^{+}$and the set of partitions such that $\ell(\lambda) \leq g$.

Let $G$ be a classical group $\operatorname{GL}(n, \mathbb{Q})$ or $\operatorname{Sp}(2 g, \mathbb{Q}), T$ its fixed maximal torus, $P$ its weight lattice and $P^{+}$the set of dominant integral weight with respect to $T$. For a rational representation $V$ of $G$, there exists an irreducible decomposition $V=$ $\bigoplus_{\lambda \in P} V_{\lambda}$ as a $T$-module where $V_{\lambda}:=\{v \in V \mid t v=\lambda(t) v$ for any $t \in T\}$. We call this decomposition a weight decomposition of $V$ with respect to $T$. If $V_{\lambda} \neq\{0\}$, then we call $\lambda$ a weight of $V$. For a weight $\lambda$, a non-zero vector $v \in V_{\lambda}$ is called a weight vector of weight $\lambda$.

Let $U$ be the subgroup of $G$ consists of all upper unitriangular matrices in $G$. For a rational representation $V$ of $G$, we define $V^{U}:=\{v \in V \mid u v=v$ for all $u \in U\}$. We call a non-zero vector $v \in V^{U}$ a maximal vector of $V$. This subspace $V^{U}$ is $T$-stable. Thus, as a $T$-module, $V^{U}$ has a irreducible decomposition $V^{U}=\bigoplus_{\lambda \in P} V_{\lambda}^{U}$, where $V_{\lambda}^{U}:=V^{U} \cap V_{\lambda}$. 


\section{Theorem 4.1 (Cartan and Weyl's highest weight theory)}

(i) Any rational representation of $V$ is completely reducible.

(ii) Suppose $V$ is an irreducible rational representation of $G$. Then $V^{U}$ is onedimensional, and the weight $\lambda$ of $V^{U}=V_{\lambda}^{U}$ belongs to $P^{+}$. We call this $\lambda$ the highest weight of $V$, and any non-zero vector $v \in V_{\lambda}^{U}$ is called a highest weight vector of $V$.

(iii) For any $\lambda \in P^{+}$, there exists a unique (up to isomorphism) irreducible rational representation $L^{\lambda}$ of $G$ with highest weight $\lambda$. Moreover, for two $\lambda, \mu \in P^{+}$, $L^{\lambda} \cong L^{\mu}$ if and only if $\lambda=\mu$.

(iv) The set of isomorphism classes of irreducible rational representations of $G$ is parametrized by the set $P^{+}$of dominant integral weights.

(v) Let $V$ be a rational representation of $G$ and $\chi_{V}$ a character of $V$ as a $T$ module. Then for two rational representation $V$ and $W$, they are isomorphic as $G$-modules if and only if $\chi_{V}=\chi_{W}$.

Remark 4.2 We can parametrize the set of isomorphism classes of irreducible rational representations of $\mathrm{GL}(n, \mathbb{Q})$ by $P_{\mathrm{GL}(n, \mathbb{Q})}^{+}$. On the other hand, we define the determinant representation by $\operatorname{det}^{e}: \operatorname{GL}(n, \mathbb{Q}) \ni X \rightarrow \operatorname{det} X^{e} \in \mathbb{Q}^{\times}$. The highest weight of this representation is given by $(e, e, \cdots, e) \in P_{\mathrm{GL}(n, \mathbb{Q})}^{+}$. If $\lambda \in P^{+}$satisfies $\lambda_{n}<0$, then $L^{\lambda} \cong \operatorname{det}^{-\lambda_{n}} \otimes L^{\left(\lambda_{1}-\lambda_{n}, \lambda_{2}-\lambda_{n}, \ldots, 0\right)}$. Moreover the set of isomorphism classes of polynomial irreducible representations is parametrized by the set of partitions $\lambda$ such that $\ell(\lambda) \leq n$. We denote the polynomial representations corresponding to a partition $\lambda$ by $L_{\mathrm{GL}}^{\lambda}, L^{(\lambda)}$ or simply $(\lambda)$.

Remark 4.3 We can parametrize the set of isomorphism classes of irreducible rational representations of $\operatorname{Sp}(2 g, \mathbb{Q})$ by

$$
P_{\mathrm{Sp}(2 g, \mathbb{Q})}^{+} \cong\left\{\lambda_{1} \geq \lambda_{2} \geq \cdots \geq \lambda_{g} \geq 0 \mid \lambda_{i} \in \mathbb{Z}, 1 \leq i \leq n\right\},
$$

namely the set of partitions $\lambda$ such that $\ell(\lambda) \leq g$. In this paper, we denote the irreducible representation corresponding to $\lambda$ by $L_{\mathrm{Sp}}^{\lambda}, L^{[\lambda]}$ or simply $[\lambda]$.

Note that the natural representation $H_{\mathbb{Q}}=\mathbb{Q}^{2 g}$ of $\operatorname{Sp}(2 g, \mathbb{Q})$ is irreducible with highest weight $(1,0, \ldots, 0)$ and $H_{\mathbb{Q}}^{*} \cong H_{\mathbb{Q}}$ by Poincaré duality. More precisely, we set $i^{\prime}:=2 g-i+1$ for each integer $1 \leq i \leq 2 g$. Then for the standard basis $\left\{e_{i}\right\}_{i=1}^{2 g}$ of $H_{\mathbb{Q}}$, we see

$$
\left\langle e_{i}, e_{j}\right\rangle=0=\left\langle e_{i^{\prime}}, e_{j^{\prime}}\right\rangle, \quad\left\langle e_{i}, e_{j^{\prime}}\right\rangle=\delta_{i j}=-\left\langle e_{j^{\prime}}, e_{i}\right\rangle, \quad(1 \leq i \leq g) .
$$


There is an isomorphism $H_{\mathbb{Q}} \rightarrow H_{\mathbb{Q}}^{*}$ as $\operatorname{Sp}(2 g, \mathbb{Q})$-modules given by

$$
H_{\mathbb{Q}} \ni v \mapsto\langle\bullet, v\rangle \in H_{\mathbb{Q}}^{*}
$$

In general, every irreducible rational representation $[\lambda]$ is isomorphic to its dual.

Let us recall Pieri's formula, the simplest version of the decomposition of tensor product representations. For two partitions $\lambda$ and $\mu$ satisfying $\lambda \supset \mu$, the skew shape $\lambda \backslash \mu$ is a vertical strip if there is at most one box in each row.

Theorem 4.4 (Pieri's formula) Let $\mu$ be a partition such that $\ell(\mu) \leq n$. Then

$$
L_{\mathrm{GL}}^{\left(1^{k}\right)} \otimes L_{\mathrm{GL}}^{\mu} \cong \bigoplus_{\lambda} L_{\mathrm{GL}}^{\lambda},
$$

where $\lambda$ runs over the set of partitions obtained by adding a vertical $k$-strip to $\mu$ such that $\ell(\lambda) \leq n$.

\subsection{Branching rules from $\operatorname{GL}(2 g, \mathbb{Q})$ to $\operatorname{Sp}(2 g, \mathbb{Q})$}

We regard $\operatorname{Sp}(2 g, \mathbb{Q})$ as a subgroup of $\mathrm{GL}(2 g, \mathbb{Q})$. We consider the restriction of an irreducible polynomial representation $L_{\mathrm{GL}}^{\lambda}$ to $\operatorname{Sp}(2 g, \mathbb{Q})$. We can give its irreducible decomposition using the Littlewood-Richardson coefficients $\operatorname{LR}_{\lambda \mu}^{v}$ as follows.

Theorem 4.5 (Fulton and Harris [6, 25.39], and Koike and Terada [16, Proposition 2.5.1]) Let $\lambda=\left(\lambda_{1} \geq \lambda_{2} \geq \cdots \geq \lambda_{g} \geq 0\right)$ be a partition such that $\ell(\lambda) \leq g$. Then we have

$$
\operatorname{Res}_{\mathrm{Sp}(2 g, \mathbb{Q})}^{\mathrm{GL}(2 g, \mathbb{Q})}\left(L_{\mathrm{GL}}^{\lambda}\right) \cong \bigoplus_{\bar{\lambda}} N_{\lambda \bar{\lambda}} L_{\mathrm{Sp}}^{\bar{\lambda}},
$$

where $\bar{\lambda}$ runs over all partitions such that $\ell(\bar{\lambda}) \leq g$. Here $N_{\lambda \bar{\lambda}}=\sum_{\eta} \operatorname{LR}_{\eta \bar{\lambda}}^{\lambda}$, where $\eta$ runs over all partitions $\eta=\left(\eta_{1}=\eta_{2} \geq \eta_{3}=\eta_{4} \geq \cdots\right)$ with each part occurring an even number of times, namely $\eta^{\prime}$ even. Here $\eta^{\prime}$ is a conjugate partition of $\eta$.

Remark 4.6 We give a combinatorial description of the Littlewood-Richardson coefficients. (See, eg, Fulton and Harris [6], and Macdonald [20].) For two Young diagrams $\lambda$ and $\mu$ satisfying $\lambda \subset \mu$, we denote by $\lambda \backslash \mu$ a skew Young diagram, which is the difference of $\lambda$ and $\mu$. For a skew Young diagram $\lambda \backslash \mu$ of size $m$, a semi-standard tableau of shape $\lambda \backslash \mu$ is an array $T$ of positive integers $1,2, \ldots, m$ of shape $\lambda \backslash \mu$ that is weakly increasing in every row and strictly increasing in every column. 
(i) For two partitions $\lambda \supset \mu$, a semi-standard tableau on $\lambda \backslash \mu$ is a numbering on $\lambda \backslash \mu \rightarrow \mathbb{Z}_{\geq 1}$ such that the numbers inserted in $\lambda \backslash \mu$ must increase strictly down each column and weakly from left to right along each row. For a semi-standard tableau on $\lambda \backslash \mu$, we denote the number of $i$ appearing in this semi-standard tableau by $m_{i}$. We call $\left(m_{1}, m_{2}, \ldots\right)$ a weight of the semi-standard tableau.

(ii) For a semi-standard tableau $T$ on $\lambda \backslash \mu$, we define a sequence $w(T)$ of integers by reading the numbers inserted in $\lambda \backslash \mu$ from right to left in successive rows, starting with top row.

(iii) For a sequence $w=\left(a_{1} a_{2} \cdots\right)$, we denote the number of $i$ appearing in a subsequence $\left(a_{1} a_{2} \cdots a_{r}\right)$ by $m_{i}\left(a_{1} a_{2} \cdots a_{r}\right)$. A sequence $w$ is a lattice permutation if $m_{1}\left(a_{1} a_{2} \cdots a_{r}\right) \geq m_{2}\left(a_{1} a_{2} \cdots a_{r}\right) \geq \cdots$ for any $r \geq 1$.

The Littlewood-Richardson coefficients $\mathrm{LR}_{\mu \nu}^{\lambda}$ is the number of semi-standard tableaux $T$ on $\lambda \backslash \mu$ with weight $v$ such that $w(T)$ is a lattice permutation.

\subsection{Review on the classical Schur-Weyl duality}

For the natural representation $H_{\mathbb{Q}} \cong L^{(1)}$ of $\operatorname{GL}(n, \mathbb{Q})$, we consider the $k^{\text {th }}$ tensor product representation $\rho_{k}: \operatorname{GL}(n, \mathbb{Q}) \rightarrow \operatorname{GL}\left(H_{\mathbb{Q}}^{\otimes k}\right)$ of $H_{\mathbb{Q}}$. For each $k \geq 1$, the symmetric group $\mathfrak{S}_{k}$ of degree $k$ naturally acts on the space $H_{\mathbb{Q}}^{\otimes k}$ from the right as a permutation of the components. Since these two actions are commutative, we can decompose $H_{\mathbb{Q}}^{\otimes k}$ as a $\left(\operatorname{GL}(n, \mathbb{Q}) \times \mathfrak{S}_{k}\right)$-module. Let us recall this irreducible decomposition, called the Schur-Weyl duality for $\operatorname{GL}(n, \mathbb{Q})$ and $\mathfrak{S}_{k}$.

Theorem 4.7 (Schur-Weyl duality for $\operatorname{GL}(n, \mathbb{Q})$ and $\left.\mathfrak{S}_{k}\right)$

(i) Let $\lambda$ be a partition of $k$ such that $\ell(\lambda) \leq n$. There exists a non-zero maximal vector $v_{\lambda}$ with weight $\lambda$ satisfying the following three conditions:

(a) The $\mathfrak{S}_{k}$-invariant subspace $S^{\lambda}:=\sum_{\sigma \in \mathfrak{S}_{k}} \mathbb{Q} v_{\lambda} \cdot \sigma$ gives an irreducible repre-
sentation of $\mathfrak{S}_{k}$.

(b) The subspace $\left(H_{\mathbb{Q}}^{\otimes k}\right)_{\lambda}^{U}$ of weight $\lambda$ coincides with the subspace $S^{\lambda}$, where $U$ is the fixed unipotent subgroup of $\operatorname{GL}(n, \mathbb{Q})$ consisting of upper unitriangular matrices.

(c) The $\operatorname{GL}(n, \mathbb{Q})$-module generated by $v_{\lambda}$ is isomorphic to the irreducible representation $L_{\mathrm{GL}}^{(\lambda)}$ of $\mathrm{GL}(n, \mathbb{Q})$ with highest weight $\lambda$.

(ii) We have the irreducible decomposition

$$
H_{\mathbb{Q}}^{\otimes k} \cong \bigoplus_{\lambda=\left(\lambda_{1} \geq \cdots \geq \lambda_{n} \geq 0\right) \vdash k} L^{\lambda} \otimes S^{\lambda}
$$

as $\left(\mathrm{GL}(n, \mathbb{Q}) \times \mathfrak{S}_{k}\right)$-modules. 
(iii) Suppose $n \geq k$. Then $\left\{S^{\lambda} \mid \lambda \vdash k\right\}$ gives a complete set of representatives of irreducible representations of $\mathfrak{S}_{k}$.

\section{Remark 4.8}

(i) The irreducible representation $S^{\lambda}$ of $\mathfrak{S}_{k}$ is isomorphic to the following $\mathfrak{S}_{k}-$ module.

For a partition $\lambda$ of $k$, we define two special Young subgroups

$$
C_{\lambda}:=\mathfrak{S}_{\lambda_{1}} \times \mathfrak{S}_{\lambda_{2}} \times \cdots \quad \text { and } \quad R_{\lambda}:=\mathfrak{S}_{\lambda_{1}^{\prime}} \times \mathfrak{S}_{\lambda_{2}^{\prime}} \times \cdots
$$

of $\mathfrak{S}_{k}$. Here a partition $\lambda^{\prime}=\left(\lambda_{1}^{\prime}, \lambda_{2}^{\prime}, \ldots\right)$ is the conjugate partition of $\lambda$. In the group algebras of these two groups, we find idempotents

$$
a_{\lambda}=\frac{1}{\left|R_{\lambda}\right|} \sum_{\sigma \in R_{\lambda}} \sigma \in \mathbb{Q} R_{\lambda} \quad \text { and } \quad b_{\lambda}=\frac{1}{\left|C_{\lambda}\right|} \sum_{\sigma \in C_{\lambda}} \operatorname{sgn}(\sigma) \sigma \in \mathbb{Q} C_{\lambda} .
$$

Then $c_{\lambda}=\left|R_{\lambda}\right|\left|C_{\lambda}\right| a_{\lambda} b_{\lambda}$ gives an idempotent in $\mathbb{Q} \mathfrak{S}_{k}$, called the Young symmetrizer for $\lambda$. The right ideal $c_{\lambda} \cdot \mathbb{Q} \mathfrak{S}_{k}$ in $\mathbb{Q} \mathfrak{S}_{k}$ gives an irreducible $\mathfrak{S}_{k}$-module that is isomorphic to $S^{\lambda}$ above.

(ii) We construct $v_{\lambda}$ appearing in the theorem above by the following way. First, we define $v_{1} \wedge v_{2} \wedge \cdots \wedge v_{r}$ to be an anti-symmetrizer

$$
\sum_{\sigma \in \mathfrak{S}_{r}} \operatorname{sgn}(\sigma)\left(v_{1} \otimes v_{2} \otimes \cdots \otimes v_{r}\right) \cdot \sigma \in H_{\mathbb{Q}}^{\otimes r}
$$

For the natural base $\left\{e_{i}\right\}_{i=1}^{n}$ of $H_{\mathbb{Q}}$, we define

$$
v_{\lambda}:=\left(e_{1} \wedge \cdots \wedge e_{\lambda_{1}^{\prime}}\right) \otimes\left(e_{1} \wedge \cdots \wedge e_{\lambda_{2}^{\prime}}\right) \otimes \cdots \in H_{\mathbb{Q}}^{\otimes k} .
$$

Note that $v_{\lambda}$ is a maximal vector of weight $\lambda$ and

$$
v_{\lambda}=\left(e_{1} \otimes \cdots \otimes e_{\lambda_{1}^{\prime}} \otimes e_{1} \otimes \cdots \otimes e_{\lambda_{2}^{\prime}} \otimes \cdots\right) \cdot c_{\lambda}
$$

This $v_{\lambda}$ gives our desirable vector in the theorem above.

\subsection{Brauer-Schur-Weyl duality}

The first two subsections are based on $\mathrm{Hu}$ and Yang [10], and $\mathrm{Hu}$ [9]. The last one is based on Ram [26]. 
4.4.1 Brauer algebras Let us define the Brauer algebra $B_{k}(-2 g)$ with a parameter $-2 g$ and size $k$.

Definition 4.9 The Brauer algebra $B_{k}(-2 g)$ over $\mathbb{Q}$ is a unital associative $\mathbb{Q}$-algebra with the following generators and defining relations:

$$
\begin{aligned}
& \text { generators : } s_{1}, \ldots, s_{k-1}, \gamma_{1}, \ldots, \gamma_{n-1} \text {, } \\
& \text { relations : } s_{i}^{2}=1, \gamma_{i}^{2}=(-2 g) \gamma_{i}, \gamma_{i} s_{i}=\gamma_{i}=s_{i} \gamma_{i} \quad(1 \leq i \leq k-1) \text {, } \\
& s_{i} s_{j}=s_{j} s_{i}, s_{i} \gamma_{j}=\gamma_{j} s_{i}, \gamma_{i} \gamma_{j}=\gamma_{j} \gamma_{i} \quad(1 \leq i<j-1 \leq k-2), \\
& s_{i} s_{i+1} s_{i}=s_{i+1} s_{i} s_{i+1}, \gamma_{i} \gamma_{i+1} \gamma_{i}=\gamma_{i}, \gamma_{i+1} \gamma_{i} \gamma_{i+1}=\gamma_{i+1} \text {, } \\
& (1 \leq i \leq k-2) \text {, } \\
& s_{i} \gamma_{i+1} \gamma_{i}=s_{i+1} \gamma_{i}, \gamma_{i+1} \gamma_{i} s_{i+1}=\gamma_{i+1} s_{i} \quad(1 \leq i \leq k-2) \text {. }
\end{aligned}
$$

Remark 4.10 The Brauer algebra $B_{k}(-2 g)$ is obtained by the following diagrammatic way.

First of all, the Brauer $k$ diagram is a diagram with $2 k$ specific vertices arranged in two rows of $k$ each, the top row and the bottom row, and exactly $k$ edges such that every vertex is joined to another vertex (distinct from itself) by exactly one edge.

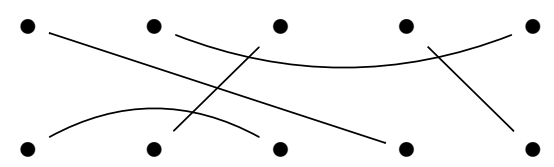

We define a multiplication of two diagrams as follows. We compose two diagrams $D_{1}$ and $D_{2}$ by identifying the bottom row of $D_{1}$ with the top row of $D_{2}$ such that the $i^{\text {th }}$ vertex in the bottom row of $D_{1}$ coincides with the $i^{\text {th }}$ vertex in the top row of $D_{2}$. The result is a graph, with a certain number, $n\left(D_{1}, D_{2}\right)$, of interior loops. After removing the interior loops and the identified vertices, retaining the edges and remaining vertices, we obtain a new Brauer $k$-diagram $D_{1} \circ D_{2}$. Then we define a multiplication $D_{1} \cdot D_{2}$ by $(-2 g)^{n\left(D_{1}, D_{2}\right)} D_{1} \circ D_{2}$.

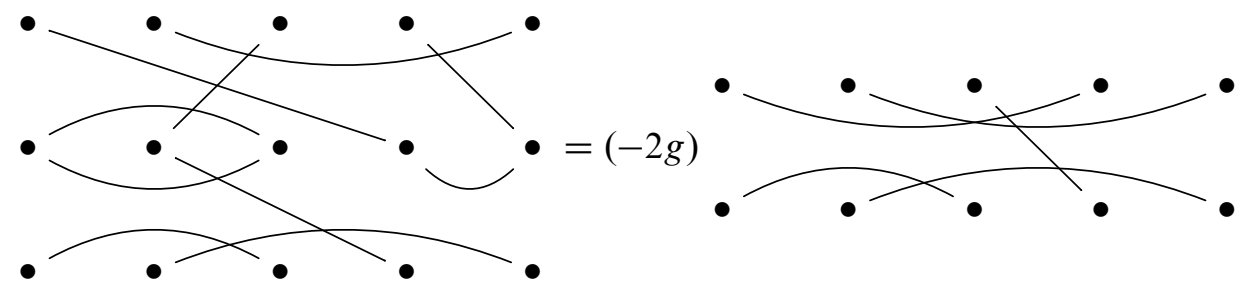


The Brauer algebra $B_{k}(-2 g)$ is defined as $\mathbb{Q}$-linear space with a basis being the set of the Brauer $k$-diagrams and the multiplication of two elements given by the linear extension of a product above.

The generators $s_{i}$ and $\gamma_{i}$ correspond to the following diagrams.

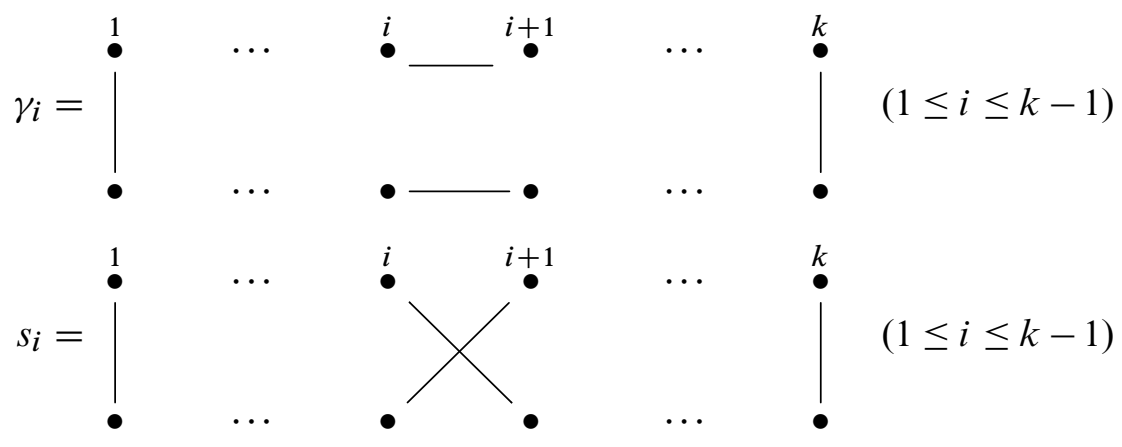

\subsubsection{Decomposition of tensor spaces (Brauer-Schur-Weyl duality) Let us recall} the inner product on $H_{\mathbb{Q}}$ defined by (3). Set $i^{\prime}:=2 g-i+1$ for each integer $1 \leq i \leq 2 g$. For the standard basis $\left\{e_{i}\right\}_{i=1}^{2 g}$ of $H_{\mathbb{Q}}$, we see

$$
\left\langle e_{i}, e_{j}\right\rangle=0=\left\langle e_{i^{\prime}}, e_{j^{\prime}}\right\rangle, \quad\left\langle e_{i}, e_{j^{\prime}}\right\rangle=\delta_{i j}=-\left\langle e_{j^{\prime}}, e_{i}\right\rangle \quad(1 \leq i \leq g) .
$$

For each integer $1 \leq i \leq 2 g$, we define

$$
e_{i}^{*}= \begin{cases}e_{i^{\prime}} & (1 \leq i \leq g), \\ -e_{i^{\prime}} & (g+1 \leq i \leq 2 g) .\end{cases}
$$

Then both of $\left\{e_{i}\right\}_{i=1}^{2 g}$ and $\left\{e_{i}^{*}\right\}_{i=1}^{2 g}$ are bases for $H_{\mathbb{Q}}$ such that one is dual to the other in the sense that $\left\langle e_{i}, e_{j}^{*}\right\rangle=\delta_{i j}$ for any $i, j$.

The following lemma is obvious, but important for generalizing the Schur-Weyl duality for $\operatorname{Sp}(2 g, \mathbb{Q})$.

Lemma 4.11 The element $\omega:=\sum_{i=1}^{2 g} e_{i} \otimes e_{i}^{*} \in H_{\mathbb{Q}}^{\otimes 2}$ is invariant under the action of $\operatorname{Sp}(2 g, \mathbb{Q})$ on $H_{\mathbb{Q}}^{\otimes 2}$.

We define a right action of $B_{k}(-2 g)$ on $H_{\mathbb{Q}}^{\otimes k}$ as follows.

Proposition 4.12 For any $i, j$, let us define

$$
\epsilon_{i j}= \begin{cases}1 & \text { if } i=j \\ -1 & \text { if } i=j^{\prime} \\ 0 & \text { otherwise }\end{cases}
$$


There is a right action of $B_{k}(-2 g)$ on $H_{\mathbb{Q}}^{\otimes k}$ that is defined on generators by

$$
\begin{aligned}
&\left(v_{i_{1}} \otimes \cdots \otimes v_{i_{k}}\right) \cdot \gamma_{j}:=\epsilon_{i_{j} i_{j+1}} v_{i_{1}} \otimes \cdots \otimes v_{i_{j-1}} \otimes\left(\sum_{r=1}^{2 g} e_{k} \otimes e_{k}^{*}\right) \\
& \otimes v_{i_{j+2}} \otimes \cdots \otimes v_{i_{k}}, \\
&\left(v_{i_{1}} \otimes \cdots \otimes v_{i_{k}}\right) \cdot s_{j}:=-v_{i_{1}} \otimes \cdots \otimes v_{i_{j-1}} \otimes v_{i_{j+1}} \otimes v_{i_{j}} \otimes v_{i_{j+2}} \otimes \cdots \otimes v_{i_{k}},
\end{aligned}
$$

for any $v_{i_{1}}, \ldots, v_{i_{k}} \in H_{\mathbb{Q}}$. Moreover, this action commutes with that of $\operatorname{Sp}(2 g, \mathbb{Q})$.

Here we state the Brauer-Schur-Weyl duality.

Theorem 4.13 (Brauer-Schur-Weyl duality for $\operatorname{Sp}(2 g, \mathbb{Q})$ and $B_{k}(-2 g)$ )

(i) Let $\lambda$ be a partition of $k-2 j$ for $0 \leq j \leq\left\lfloor\frac{k}{2}\right\rfloor$ such that $\ell(\lambda) \leq g$. Then there exists a maximal vector $v_{\lambda} \in H_{\mathbb{Q}}^{\otimes k}$ with highest weight $\lambda$ satisfying the following three conditions:

(a) $A B_{k}(-2 g)$-submodule

$$
D^{\lambda}:=\sum_{\sigma \in B_{k}(-2 g)} \mathbb{Q} v_{\lambda} \cdot \sigma
$$

of $H_{\mathbb{Q}}^{\otimes k}$ gives an irreducible representation of $B_{k}(-2 g)$.

(b) The subspace $\left(H_{\mathbb{Q}}^{\otimes k}\right)_{\lambda}^{U}$ of $H_{\mathbb{Q}}^{\otimes k}$ coincides with $D^{\lambda}$. Here $U$ is the fixed unipotent subgroup for $\operatorname{Sp}(2 g, \mathbb{Q})$.

(c) The $\operatorname{Sp}(2 g, \mathbb{Q})$-module generated by $v_{\lambda}$ is isomorphic to the irreducible representation $L_{\mathrm{Sp}}^{[\lambda]}$ of $\operatorname{Sp}(2 g, \mathbb{Q})$ with highest weight $\lambda$.

(ii) We have the irreducible decomposition

$$
H_{\mathbb{Q}}^{\otimes k} \cong \bigoplus_{j=0}^{\left\lfloor\frac{k}{2}\right\rfloor} \bigoplus_{\lambda \vdash k-2 j, \ell(\lambda) \leq g} L_{\mathrm{Sp}}^{[\lambda]} \otimes D^{\lambda} .
$$

as an $\left(\operatorname{Sp}(2 g, \mathbb{Q}) \times B_{k}(-2 g)\right)-$ module.

(iii) Suppose $g \geq k$. Then $\left\{D^{\lambda} \mid \lambda \vdash k-2 j\left(0 \leq j \leq\left\lfloor\frac{k}{2}\right\rfloor\right)\right\}$ gives a complete set of representatives of irreducible representations of $B_{k}(-2 g)$.

In order to show our main theorem, it is important to observe an explicit construction of $v_{\lambda}$ and a description of $D^{\lambda}$. By combining the following theorem with some results explained in the next section, we obtain a description of the Sp-maximal vectors in $\mathfrak{h}_{g, 1}^{\mathbb{Q}}(k)$. Thus we can get some series of Sp-irreducible representations in the Johnson cokernels systematically by using a strategy described in Proposition 7.1 and a subsequent remark. 
Theorem 4.14 [9, Definition 3.9, Lemma 3.10, Lemma 4.8]

(i) For a partition $\lambda$ of $k-2 j$ for $0 \leq j \leq\left\lfloor\frac{k}{2}\right\rfloor$ such that $\ell(\lambda) \leq g$, a maximal vector $v_{\lambda}$ is given by $v_{\lambda}:=\omega^{\otimes j} \otimes\left(e_{1} \wedge \cdots \wedge e_{\lambda_{1}^{\prime}}\right) \otimes\left(e_{1} \wedge \cdots \wedge e_{\lambda_{2}^{\prime}}\right) \otimes \cdots$.

(ii) We regard a subalgebra generated by $s_{i}(1 \leq i \leq k-1)$ in $B_{k}(-2 g)$ as a group algebra $\mathbb{Q} \mathfrak{S}_{k}$. Then the right module $v_{\lambda} \cdot B_{k}(-2 g)$ coincides with $v_{\lambda} \cdot \mathbb{Q} \mathfrak{S}_{k}$ as a $\mathbb{Q}$-vector space.

\subsubsection{Character values and decompositions of $\boldsymbol{D}^{\boldsymbol{\lambda}}$ as an $\mathfrak{S}_{\boldsymbol{k}}$-module We give} a branching law of the irreducible $B_{k}(-2 g)$-modules $D^{\lambda}$ as $\mathfrak{S}_{k}$-modules. But confusingly, the algebra $\mathbb{Q S}_{k}$ has an involution $\iota: \sigma \mapsto \operatorname{sgn}(\sigma) \sigma$, and the action of a subalgebra generated by $s_{i}$ 's in $B_{k}(-2 g)$ on $H_{\mathbb{Q}}^{\otimes k}$ is twisted by this involution. Therefore a $\mathbb{Q} \mathfrak{S}_{k}$-module $D$ is isomorphic to $\mathbf{s g n} \otimes D$ as an $\iota\left(\mathbb{Q} \mathfrak{S}_{k}\right)$-module. Here sgn is the signature representation of $\mathfrak{S}_{k}$. Note that an irreducible $\mathfrak{S}_{k}$-module $S^{\nu^{\prime}}$ is isomorphic to $\mathbf{s g n} \otimes S^{v}$.

For our purposes, we consider the ordinary (untwisted) action of $\mathfrak{S}_{k}$ on $H_{\mathbb{Q}}^{\otimes k}$ in the following theorem (ii).

\section{Theorem 4.15 [26, Theorem 5.1]}

(i) For a partition $\lambda$ of $k-2 j$ for $0 \leq j \leq\left\lfloor\frac{k}{2}\right\rfloor$ such that $\ell(\lambda) \leq g$, let $\chi_{B_{k}(-2 g)}^{\lambda}$ be the irreducible character of $D^{\lambda}$. Then we have

$$
\chi_{B_{k}(-2 g)}^{\lambda}(\sigma)=\sum_{\nu \vdash k, v \supset \lambda^{\prime}}\left(\sum_{\beta \text { even }} \operatorname{LR}_{\lambda^{\prime} \beta}^{v}\right) \chi_{\mathfrak{S}_{k}}^{\nu}(\sigma)
$$

for any $\sigma \in \mathfrak{S}_{k} \subset$ (a subalgebra generated by $\left\{s_{i}\right\}_{i=1}^{k-1}$ ). Here $\chi_{\mathfrak{S}_{k}}^{v}$ is an irreducible character of $\mathfrak{S}_{k}$ associated to a partition $v$ of $k$. The number LR is the Littlewood-Richardson coefficient. The even partition $\beta=\left(\beta_{1}, \beta_{2}, \ldots\right)$ is a partition such that any parts $\beta_{i}$ are even.

(ii) We have that the irreducible decomposition of $D^{\lambda}$ is given by

$$
\bigoplus_{\nu \vdash k, \nu \supset \lambda^{\prime}}\left(S^{\nu^{\prime}}\right)^{\oplus \sum_{\beta \text { even }} \mathrm{LR}_{\lambda^{\prime} \beta}^{\nu^{\prime}}}
$$

with respect to the ordinary $\mathfrak{S}_{k}$-action on $H_{\mathbb{Q}}^{\otimes k}$.

Remark 4.16 For a partition $\lambda \vdash k-2 j$, we have the following dimension formula:

$$
\operatorname{dim} D^{\lambda}={ }_{k} C_{2 j}(2 j-1) ! ! \cdot \operatorname{dim} S^{\lambda}
$$

This gives the multiplicity of $L_{\mathrm{Sp}}^{\lambda}$ in $H_{\mathbb{Q}}^{\otimes k}$. For $\lambda=0$, the formula above is nothing but [23, Lemma 4.1]. 


\section{Dynkin-Specht-Wever idempotent and the free Lie alge- bras}

In this section, we give a characterization of elements in $\mathfrak{h}_{g, 1}^{\mathbb{Q}}(k)$ by using two specific idempotents in the group ring of the symmetric group. The first one is the DynkinSpecht-Wever idempotent. For an element $v \in H_{\mathbb{Q}}^{\otimes k}$, this idempotent gives a necessary and sufficient condition for $v$ to be contained in the free Lie algebra $\mathcal{L}_{2 g}^{\mathbb{Q}}(k)$. The other is $1+\sigma_{k}+\sigma_{k}^{2}+\cdots+\sigma_{k}^{k-1} \in \mathbb{Q} \mathfrak{S}_{k}$, where $\sigma_{k}$ is the cyclic permutation of length $k$ defined by $\sigma_{k}(i)=i+1(1 \leq i \leq k-1)$ and $\sigma_{k}(k)=1$. By using these two idempotents we obtain a characterization of elements in $\mathfrak{h}_{g, 1}^{\mathbb{Q}}(k)$ in Corollary 5.3. In Section 7.3, we use this corollary to describe Sp-maximal vectors in $\mathfrak{h}_{g, 1}^{\mathbb{Q}}(k)$ with Theorem 4.14 .

Let us consider the right action of $\mathfrak{S}_{k+2}$ on $H_{\mathbb{Q}}^{\otimes(k+2)}$. Set $\sigma_{i}:=s_{i-1} s_{i-2} \cdots s_{1}$ for each $2 \leq i \leq k+2$, and

$$
\theta_{k+2}:=\left(1-\sigma_{2}\right) \cdots\left(1-\sigma_{k+2}\right) \in \mathbb{Q S}_{k+2} .
$$

This element characterizes the degree $(k+2)^{\text {nd }}$ part $\mathcal{L}_{2 g}^{\mathbb{Q}}(k+2)$ of the free Lie algebra $\mathcal{L}_{2 g}^{\mathbb{Q}}$ generated by $H_{\mathbb{Q}}=\mathbb{Q}^{2 g}$ as follows. (See, eg, Garsia [7, Theorem 2.1], Reutenauer [27, Theorem 8.16], and Morita [23, Lemma 4.5].)

\section{Theorem 5.1 (Dynkin-Specht-Wever)}

(i) $\theta_{k+2}^{2}=(k+2) \theta_{k+2}$. We call an element $(1 / k+2) \theta_{k+2}$ the Dynkin-SpechtWever idempotent.

(ii) For $v_{1} \otimes v_{2} \otimes \cdots \otimes v_{k+2} \in H_{\mathbb{Q}}^{\otimes k+2}$, a left-normed element $\left[v_{1}, v_{2}, \ldots, v_{k+2}\right] \in$ $\mathcal{L}_{2 g}^{\mathbb{Q}}(k+1)$ coincides with $\left(v_{1} \otimes v_{2} \otimes \cdots \otimes v_{k+2}\right) \cdot \theta_{k+2}$. Hence the right action of $\theta_{k+2}$ on $H_{\mathbb{Q}}^{\otimes k+2}$ induces a projection

$$
H_{\mathbb{Q}}^{\otimes k+2} \rightarrow \mathcal{L}_{2 g}^{\mathbb{Q}}(k+1),
$$

and $H_{\mathbb{Q}}^{\otimes k+2} \cdot \theta_{k+2}$ is isomorphic to $\mathcal{L}_{2 g}^{\mathbb{Q}}(k+2)$.

(iii) For $v \in H_{\mathbb{Q}}^{\otimes(k+2)}$, the following two conditions are equivalent:

(a) $v \in \mathcal{L}_{2 g}^{\mathbb{Q}}(k+2)$

(b) $v \cdot \theta_{k+2}=(k+2) v$

Recall that we need to consider the $\operatorname{Sp}(2 g, \mathbb{Q})$-module

$$
\mathfrak{h}_{g, 1}^{\mathbb{Q}}(k)=\operatorname{Ker}\left(H_{\mathbb{Q}} \otimes_{\mathbb{Q}} \mathcal{L}_{2 g}^{\mathbb{Q}}(k+1) \rightarrow \mathcal{L}_{2 g}^{\mathbb{Q}}(k+2)\right) .
$$


To characterize $\mathfrak{h}_{g, 1}^{\mathbb{Q}}(k)$ in $H_{\mathbb{Q}}^{\otimes k+2}$, let us consider the subgroup $P$ of $\mathfrak{S}_{k+2}$ that fixes 1. Namely, $P$ is isomorphic to $\mathfrak{S}_{k+1}$. Set

$$
\theta_{P}:=\left(1-s_{2}\right)\left(1-s_{3} s_{2}\right) \cdots\left(1-s_{k+1} s_{k} \cdots s_{2}\right) .
$$

We can regard this element in $\mathbb{Q} P$ as the Dynkin-Specht-Wever idempotent for $P$. Using this element, we obtain a characterization of $\mathfrak{h}_{g, 1}^{\mathbb{Q}}(k)$ as the following theorem.

Proposition 5.2 [23, Proposition 4.6] For $v \in H_{\mathbb{Q}}^{\otimes(k+2)}$, the following two conditions are equivalent:

(i) $v \in \mathfrak{h}_{g, 1}^{\mathbb{Q}}(k)$

(ii) $v \cdot \theta_{P}=(k+1) v$ and $v \cdot \sigma_{k+2}=v$

Corollary 5.3 We have

$\theta_{P} \cdot\left(1+\sigma_{k+2}+\sigma_{k+2}^{2}+\cdots+\sigma_{k+2}^{k+1}\right) \cdot \theta_{P}=(k+1) \theta_{P} \cdot\left(1+\sigma_{k+2}+\sigma_{k+2}^{2}+\cdots+\sigma_{k+2}^{k+1}\right)$ on $H_{\mathbb{Q}}^{\otimes k+2}$. Thus we obtain

$$
v \cdot \theta_{P}\left(1+\sigma_{k+2}+\sigma_{k+2}^{2}+\cdots+\sigma_{k+2}^{k+1}\right) \in \mathfrak{h}_{g, 1}^{\mathbb{Q}}(k)
$$

for any $v \in H_{\mathbb{Q}}^{\otimes k+2}$.

Proof Let us recall the following expansions of a left-normed element in the free Lie algebra:

$$
\left[x_{1}, x_{2}, \ldots, x_{m}\right]=\sum(-1)^{r} x_{i_{1}} \otimes \cdots \otimes x_{i_{r}} \otimes x_{1} \otimes x_{j_{1}} \otimes \cdots \otimes x_{j_{m-r}-1},
$$

where the sum runs over all integers $r$ and tuples $\left(i_{1}, \ldots, i_{r}\right)$ and $\left(j_{1}, \ldots, j_{m-r-1}\right)$ of integers satisfying the conditions

$$
0 \leq r \leq m-1, \quad m \geq i_{1}>\cdots>i_{r} \geq 2, \quad 2 \leq j_{1}<\cdots<j_{m-r-1} \leq m .
$$

(See eg, [27, Lemma 1.1].) The expansion above is equivalent to

$$
\sum(-1)^{r-1} x_{i_{1}} \otimes \cdots \otimes x_{i_{r}} \otimes x_{2} \otimes x_{j_{1}} \otimes \cdots \otimes x_{j_{m-r-1}},
$$

where the sum runs over all integers $r$ and tuples $\left(i_{1}, \ldots, i_{r}\right)$ and $\left(j_{1}, \ldots, j_{m-r-1}\right)$ of integers satisfying the conditions

$$
0 \leq r \leq m-1, \quad m \geq i_{1}>\cdots>i_{r} \geq 1, \quad 1 \leq j_{1}<\cdots<j_{m-r-1} \leq m
$$

and $i_{1}, \ldots, i_{r}, j_{1}, \ldots, j_{m-r-1} \neq 2$. 
Note that $\left(v_{1} \otimes \cdots \otimes v_{k+2}\right) \cdot \theta_{P}=v_{1} \otimes\left[v_{2}, \ldots, v_{k+2}\right]$ for any $v_{1}, \ldots, v_{k+2} \in H_{\mathbb{Q}}$. To prove our statement, we shall prove that

$$
\begin{aligned}
\left(v_{1} \otimes \cdots \otimes\right. & \left.v_{k+2}\right) \cdot \theta_{P} \cdot\left(1+\sigma+\cdots+\sigma^{k+1}\right) \\
= & v_{1} \otimes\left[v_{2}, \ldots, v_{k+2}\right] \\
& \quad-\sum_{j=2}^{k+2} v_{j} \otimes\left[\left[v_{2}, v_{3}, \ldots, v_{j-1}\right],\left[v_{j+1},\left[v_{j+2}, \ldots,\left[v_{k+2}, v_{1}\right] \cdots\right]\right]\right]
\end{aligned}
$$

In the formula above, the righthand side is contained in $H_{\mathbb{Q}} \otimes_{\mathbb{Q}} \mathcal{L}_{2 g}^{\mathbb{Q}}(k+1)$. Therefore if (9) is true, by Theorem 5.1, we obtain our claim.

To prove the formula (9), we set

$$
x_{1}=\left[v_{1}, \ldots, v_{j-1}\right], \quad x_{2}=v_{j}, \quad x_{3}=v_{j+1}, \ldots, \quad x_{k+4-s}=v_{k+2} .
$$

Then applying the formula (8), we expand $\left(v_{1} \otimes \cdots \otimes v_{k+2}\right) \cdot \theta_{P}$ as

$$
v_{1} \otimes \sum(-1)^{r-1} x_{i_{1}} \otimes \cdots \otimes x_{i_{r}} \otimes x_{2} \otimes x_{j_{1}} \otimes \cdots \otimes x_{j_{k+3-s-r}}
$$

satisfying a similar condition to (8). Hence, in $\left(v_{1} \otimes \cdots \otimes v_{k+2}\right) \cdot \theta_{P} \cdot\left(1+\sigma+\cdots+\sigma^{k+1}\right)$, the terms whose first part is equal to $v_{j}$ are given by

$$
v_{j} \otimes \sum(-1)^{r-1} x_{j_{1}} \otimes \cdots \otimes x_{j_{k+3-s-r}} \otimes v_{1} \otimes x_{i_{1}} \otimes \cdots \otimes x_{i_{r}}
$$

satisfying the conditions

$$
0 \leq r \leq k+3-s, \quad 1 \leq j_{1}<\cdots<j_{k+3-s-r} \leq k+2, \quad k+2 \geq i_{1}>\cdots>i_{r} \geq 1
$$

and $i_{1}, \ldots, i_{r}, j_{1}, \ldots, j_{k+3-s-r} \neq 2$.

On the other hand, note that we have the following expansion of a right-normed element in a free Lie algebra:

$\left[x_{1},\left[x_{2}, \ldots,\left[x_{m-1}, x_{m}\right] \cdots\right]\right]=\sum(-1)^{r} x_{j_{1}} \otimes \cdots \otimes x_{j_{m-r}-1} \otimes x_{m} \otimes x_{i_{1}} \otimes \cdots \otimes x_{i_{r}}$, where the sum runs over all integers $r$, tuples $\left(i_{1}, \ldots, i_{r}\right)$ and $\left(j_{1}, \ldots, j_{m-r-1}\right)$ of integers satisfying the conditions

$$
0 \leq r \leq m-1, \quad m \geq i_{1}>\cdots>i_{r} \geq 1, \quad 1 \leq j_{1}<\cdots<j_{m-r-1} \leq m .
$$

Applying this formula to (10), we obtain $-v_{j} \otimes\left[x_{1},\left[x_{2}, \ldots,\left[x_{k+4-s}, v_{1}\right]\right]\right]$ for $x_{1}=$ $\left[v_{1}, \ldots, v_{j-1}\right], x_{2}=v_{j}, x_{3}=v_{j+1}, \ldots, x_{k+4-s}=v_{k+2}$. Thus we have (9). 


\section{Multiplicities in $\operatorname{Res}_{\mathrm{Cyc}_{k}}^{\mathfrak{S}_{k}} S^{\lambda}$ via Kraśkiewicz and Weyman's combinatorial description}

To calculate the multiplicities of the Sp-irreducible representations $L_{\mathrm{Sp}}^{[k]}$ and $L_{\mathrm{Sp}}^{\left[1^{k}\right]}$ in $\mathfrak{h}_{g, 1}^{\mathbb{Q}}(k)$ and $\mathcal{C}_{2 g}^{\mathbb{Q}}(k)$, we use some multiplicity formulae for some GL-irreducible representations in $\mathfrak{h}_{g, 1}^{\mathbb{Q}}(k)$ and $\mathcal{C}_{2 g}^{\mathbb{Q}}(k)$, and the branching rules from GL to Sp. In this section, we give a combinatorial description of the multiplicities of some GLirreducible representations in $\mathcal{L}_{2 g}^{\mathbb{Q}}(k)$ and $\mathcal{C}_{2 g}^{\mathbb{Q}}(k)$.

Let $\mathrm{Cyc}_{k}$ be a cyclic group of order $k$. Take a generator $\sigma_{k}$ of $\mathrm{Cyc}_{k}$ and a primitive $k^{\text {th }}$ root $\zeta_{k} \in \mathbb{C}$ of unity. In this section, we consider representations of the cyclic group $\mathrm{Cyc}_{k}$ over an intermediate field $\mathbb{Q}\left(\zeta_{k}\right) \subset \mathbb{K} \subset \mathbb{C}$. To begin with, we define one-dimensional representations (or characters) $\chi_{k}^{j}: \mathrm{Cyc}_{k} \rightarrow \mathbb{K}^{\times}$by $\chi_{k}^{j}\left(\sigma_{k}\right)=\zeta_{k}^{j}$ for $0 \leq j \leq k-1$. Especially, we denote the trivial representation $\chi_{k}^{0}$ by $\operatorname{triv}_{k}$. The set of isomorphism classes of irreducible representations of $\mathrm{Cyc}_{k}$ is given by $\left\{\chi_{k}^{j}, 0 \leq\right.$ $j \leq k-1\}$. Consider $\mathrm{Cyc}_{k}$ as a subgroup of $\mathfrak{S}_{k}$ by an embedding $\sigma_{k}^{i} \mapsto(12 \cdots k)^{i}$ for $0 \leq i \leq k-1$. Let us recall the following proposition.

Proposition 6.1 ([5, Proposition 4.1, 4.3], Kljačko [15]) Suppose $2 g \geq k$. For a partition $\lambda$ of $k$, we have the following multiplicity formulae:

$$
\begin{aligned}
& {\left[\mathcal{C}_{2 g}^{\mathbb{Q}}(k): L_{\mathrm{GL}}^{\lambda}\right]=\left[\operatorname{Res}_{\mathrm{Cyc}_{k}}^{\mathfrak{S}_{k}} S^{\lambda}: \operatorname{triv}_{k}\right]} \\
& {\left[\mathcal{L}_{2 g}^{\mathbb{Q}}(k): L_{\mathrm{GL}}^{\lambda}\right]=\left[\operatorname{Res}_{\mathrm{Cyc}_{k}}^{\mathfrak{S}_{k}} S^{\lambda}: \chi_{k}^{1}\right]}
\end{aligned}
$$

We explain a combinatorial description of the righthand side of the above equations: Kraśkiewicz and Weyman's combinatorial description for the branching rules of irreducible $\mathfrak{S}_{k}$-modules $S^{\lambda}$ to the cyclic subgroup $\mathrm{Cyc}_{k}$. To do this, first we define a major index of a standard tableau.

Definition 6.2 For a standard tableau $T$, we define the descent set of $T$ to be the set of entries $i$ in $T$ such that $i+1$ is located in a lower row than that which $i$ is located. We denote by $D(T)$ the descent set of $T$. The major index of $T$ is defined by

$$
\operatorname{maj}(T):=\sum_{i \in D(T)} i
$$

If $D(T)=\varnothing$, we set $\operatorname{maj}(T)=0$.

Theorem 6.3 (Kraśkiewicz and Weyman [19], Reutenauer [27, Theorem 8.8, 8.9], Garsia [7, Theorem 8.4]) The multiplicity of $\chi_{k}^{j}$ in $\operatorname{Res}_{\mathrm{Cyc}_{k}}^{\mathfrak{S}_{k}} S^{\lambda}$ is equal to the number of standard tableaux with shape $\lambda$ satisfying maj $(T) \equiv j$ modulo $k$. 
Example 6.4 For $m \geq 2$, we have the following table on the multiplicities of $\operatorname{triv}_{m}=$ $\chi_{j}^{0}$ and $\chi_{j}^{1}$.

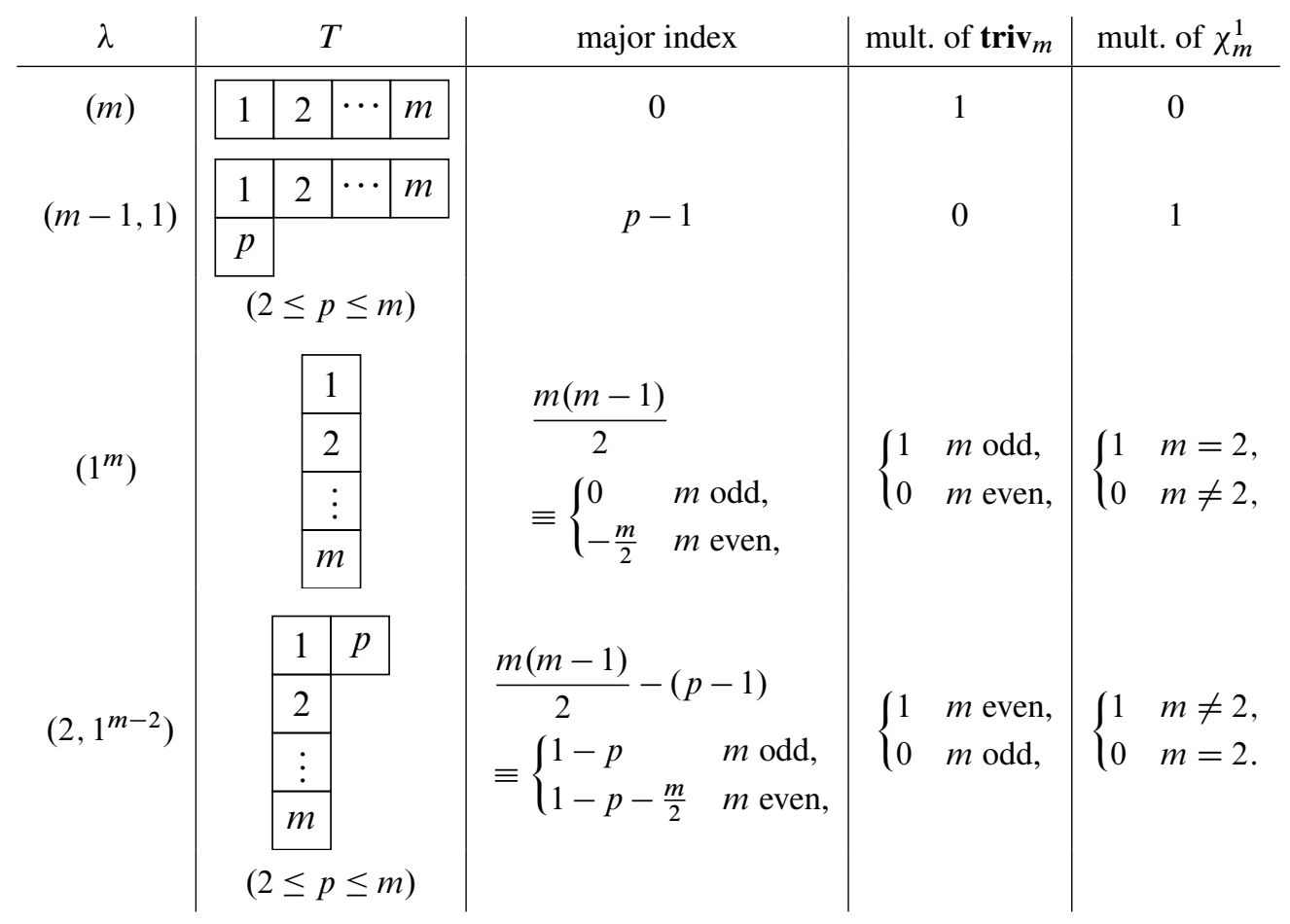

Example 6.5 For $m \geq 3$ and a partition $\lambda=\left(m-2,1^{2}\right)$, we have

(i) $\left[\operatorname{Res}_{\mathrm{Cyc}_{m}}^{\mathfrak{S}_{m}} S^{\lambda}: \operatorname{triv}_{m}\right]= \begin{cases}(m-2) / 2 & \text { if } m \text { even, } \\ (m-1) / 2 & \text { if } m \text { odd. }\end{cases}$

(ii) $\left[\operatorname{Res}_{\mathrm{Cyc}_{m}}^{\mathfrak{S}_{m}} S^{\lambda}: \chi_{m}^{1}\right]= \begin{cases}(m-3) / 2 & \text { if } m \text { odd, } \\ (m-2) / 2 & \text { if } m \text { even. }\end{cases}$

In fact, for a partition

$$
T=\begin{array}{|l|l|l|l|}
\hline 1 & 2 & \cdots & m \\
\hline p & \multicolumn{3}{|l}{} \\
\cline { 1 - 1 } q & \multicolumn{3}{|l}{} \\
\cline { 1 - 1 } & &
\end{array}
$$

its major index is given by $\operatorname{maj}(T)=p+q-2$ for $2 \leq p<q \leq m$. Then $\operatorname{maj}(T) \equiv$ $0(\bmod m)$ if and only if $p+q=m+2$. Hence we have the number of standard tableaux of shape $\lambda$ is equal to $\frac{m}{2}-1$ for odd $m$ and $\frac{m-1}{2}$ for even $m$. On the other hand, $\operatorname{maj}(T) \equiv 1(\bmod m)$ if and only if $p+q=m+3$. Hence the number of standard tableaux of shape $\lambda$ is equal to $\frac{m-3}{2}$ for odd $m$ and $\frac{m-2}{2}$ for even $m$. 
Example 6.6 For $m \geq 4$ and a partition $\lambda=\left(2^{2}, 1^{m-4}\right)$, we have

$$
\left[\operatorname{Res}_{\mathrm{Cyc}_{m}}^{\mathfrak{S}_{m}} S^{\lambda}: \chi_{m}^{1}\right]= \begin{cases}\frac{m-3}{2} & \text { if } m \text { is odd, } \\ \frac{m-4}{2} & \text { if } m \equiv 0(\bmod 4), \\ \frac{m-2}{2} & \text { if } m \equiv 2(\bmod 4) .\end{cases}
$$

To prove this, we consider the following two kinds of standard tableaux of shape $\lambda$ :

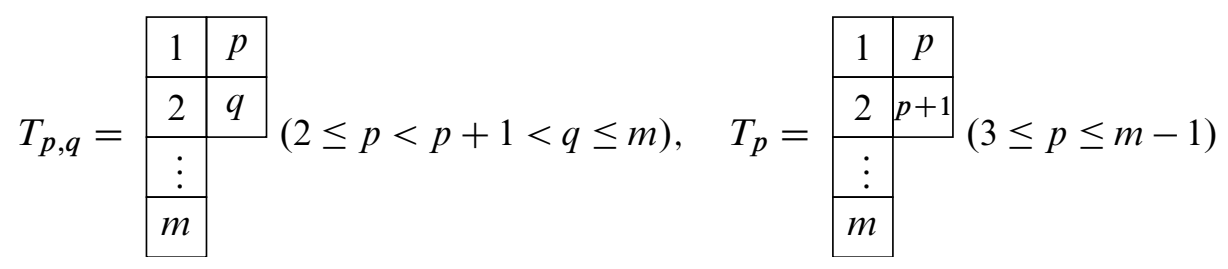

Their major indices are given by

$$
\operatorname{maj}\left(T_{p, q}\right)=\frac{m(m-1)}{2}+2-p-q \quad \text { and } \quad \operatorname{maj}\left(T_{p}\right)=\frac{m(m-1)}{2}+1-p .
$$

If $m$ is odd, $m(m-1) / 2 \equiv 0(\bmod m)$. Thus $\operatorname{maj}\left(T_{p, q}\right) \equiv 1(\bmod m)$ if and only if $p+q=m+1$. The number of such pairs $(p, q)$ is $(m-3) / 2$. There is no $T_{p}$ such that $\operatorname{maj}\left(T_{p}\right) \equiv 1(\bmod m)$. If $m$ is even, $m(m-1) / 2 \equiv m / 2(\bmod m)$. Since $m \neq 2, \operatorname{maj}\left(T_{p}\right) \equiv 1(\bmod m)$ if and only if $p=m / 2$ for $m>4$. If $m=4$, there is no such $T_{p}$.

On the other hand, $\operatorname{maj}\left(T_{p, q}\right) \equiv 1(\bmod m)$ if and only if $p+q=m+1+(m / 2)$ for $m=4,6,8$ and $p+q=m+1+(m / 2)$, or $1+(m / 2)$ for $m \geq 10$. If $m=4$, 6 or 8 , the number of such pairs $(p, q)$ is 0,1 or 1 respectively. Suppose $m \geq 10$. If $m=4 M, \operatorname{maj}\left(T_{p, q}\right) \equiv 1(\bmod m)$ if and only if $p+q=6 M+1$ or $2 M+1$. The number of such pairs $(p, q)$ is $(M-1)+(M-2)=2 M-3=(m / 2)-3$. If $m=4 M+2, \operatorname{maj}\left(T_{p, q}\right) \equiv 1(\bmod m)$ if and only if $p+q=6 M+4$ or $2 M+2$. The number of such pairs $(p, q)$ is $M+(M-1)=2 M-1=(m / 2)-2$. Therefore we obtain the claim.

\section{Sp-irreducible components of the Johnson cokernels}

This section is the main part of this paper. First, in Section 7.1, we introduce an Sp-equivariant homomorphism $c_{k}: \mathfrak{h}_{g, 1}^{\mathbb{Q}}(k) \rightarrow \mathcal{C}_{2 g}^{\mathbb{Q}}(k)$, and give a strategy to detect $\mathrm{Sp}$-irreducible representations in the Johnson cokernels. In Section 7.2, we calculate the multiplicities of $L_{\mathrm{Sp}}^{[k]}$ and $L_{\mathrm{Sp}}^{\left[1^{k}\right]}$ in $\mathfrak{h}_{g, 1}^{\mathbb{Q}}(k)$ explicitly. We give their maximal vectors explicitly in Section 7.3, and show that they do not vanish under $c_{k}$ in Section 7.4. By these results, we detect $L_{\mathrm{Sp}}^{[k]}$ for odd $k \geq 3$ and $L_{\mathrm{Sp}}^{\left[1^{k}\right]}$ for $k \geq 5$ such that $k \equiv 1(\bmod 4)$ in the Johnson cokernels. In the final subsection, we summarize our new classes of 
Johnson cokernels, and give some discussions about relationships between our classes and recent results of Conant, Kassabov and Vogtmann.

\subsection{Our strategy for detecting Sp-irreducible components}

In the rest of this paper, we assume $g \geq k+2$. To explain our strategy for detecting $\mathrm{Sp}$-irreducible components in the Johnson cokernel of the mapping class group, let us recall the following diagram as mentioned in the end of Section 3:

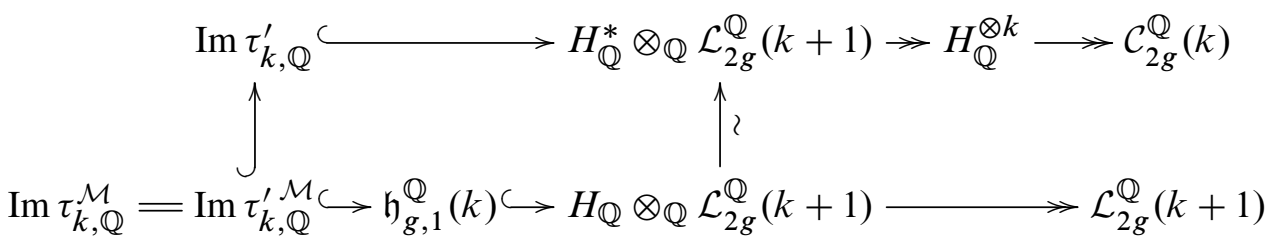

Here we may regard it as a diagram of $\operatorname{Sp}(2 g, \mathbb{Q})$-modules and $\operatorname{Sp}(2 g, \mathbb{Q})$-equivariant homomorphisms. By Theorem 3.4, we see Coker $\left(\operatorname{Im} \tau_{k, \mathbb{Q}}^{\prime} \hookrightarrow H_{\mathbb{Q}}^{*} \otimes_{\mathbb{Q}} \mathcal{L}_{2 g}^{\mathbb{Q}}(k+1)\right)$ coincides with $\mathcal{C}_{2 g}^{\mathbb{Q}}(k)$ for $2 g \geq k+2$. Observing a natural isomorphism

$$
H^{*} \otimes_{\mathbb{Q}} \mathcal{L}_{2 g}^{\mathbb{Q}}(k+1) \cong H \otimes_{\mathbb{Q}} \mathcal{L}_{2 g}^{\mathbb{Q}}(k+1)
$$

induced from the Poincare duality, we obtain $\operatorname{Sp}(2 g, \mathbb{Q})$--equivariant homomorphisms $c_{k}: \mathfrak{h}_{g, 1}^{\mathbb{Q}}(k) \rightarrow \mathcal{C}_{2 g}^{\mathbb{Q}}(k)$. Note that $\operatorname{Im} \tau_{k, \mathbb{Q}}^{\prime \mathcal{M}} \subset \operatorname{Im} \tau_{k, \mathbb{Q}}^{\prime}$. Then we have the following criterion for detecting Sp-irreducible components in the Johnson cokernel

$$
\operatorname{Coker}\left(\operatorname{Im} \tau_{k, \mathbb{Q}}^{\prime \mathcal{M}} \rightarrow \mathfrak{h}_{g, 1}^{\mathbb{Q}}(k)\right) .
$$

Proposition 7.1 Let $V$ be an irreducible $\operatorname{Sp}(2 g, \mathbb{Q})$-submodule of $\mathfrak{h}_{g, 1}^{\mathbb{Q}}(k)$. If $c_{k}(V)$ is a non-trivial (then automatically irreducible) component of $\mathcal{C}_{2 g}^{\mathbb{Q}}(k)$, then $V$ is an irreducible $\operatorname{Sp}(2 g, \mathbb{Q})$-module in $\operatorname{Coker}\left(\operatorname{Im} \tau_{k, \mathbb{Q}}^{\prime \mathcal{M}}\right)$. In particular, if there is a maximal vector $v$ of weight $\lambda$ in $\mathfrak{h}_{g, 1}^{\mathbb{Q}}(k)$ such that $c_{k}(v) \neq 0$ (then $c_{k}(v)$ is a maximal in $\left.\mathcal{C}_{2 g}^{\mathbb{Q}}(k)\right)$, then $v$ gives an $\operatorname{Sp}(2 g, \mathbb{Q})$-irreducible component in $\operatorname{Coker}\left(\operatorname{Im} \tau_{k, \mathbb{Q}}^{\prime \mathcal{M}}\right)$ that is isomorphic to the irreducible $\operatorname{Sp}(2 g, \mathbb{Q})$-module $L_{\mathrm{Sp}}^{[\lambda]}$.

To find such a maximal vector, we use Theorem 4.14 and Corollary 5.3. Namely, for a maximal vector $v_{\lambda}$ as in Theorem 4.14, we consider

$$
\phi_{\lambda}:=v_{\lambda} \cdot \theta_{P} \cdot\left(1+\sigma_{k+2}+\cdots+\sigma_{k+1}^{k+2}\right) .
$$

If $\phi_{\lambda} \neq 0$, this is a maximal vector of weight $\lambda$ such that $\phi_{\lambda} \in \mathfrak{h}_{g, 1}^{\mathbb{Q}}(k)$ by Corollary 5.3. Then we investigate whether $c_{k}\left(\phi_{\lambda}\right) \in \mathcal{C}_{2 g}^{\mathbb{Q}}(k)$ is 0 or not. 


\subsection{Some multiplicity formulae}

In this subsection, we give some explicit multiplicity formulae for $[k]$ and $\left[1^{k}\right]$ in $\mathfrak{h}_{g, 1}^{\mathbb{Q}}(k)$ and $\mathcal{C}_{2 g}^{\mathbb{Q}}(k)$. First, let us recall Proposition 6.1 and the following lemma obtained by Pieri's formula (Theorem 4.4).

Lemma 7.2 Suppose $n \geq k+2$. For a partition $\lambda$ of $k+2$,

$$
\left[H_{\mathbb{Q}} \otimes_{\mathbb{Q}} \mathcal{L}_{n}^{\mathbb{Q}}(k+1): L_{\mathrm{GL}}^{\lambda}\right]=\sum_{\mu}\left[\mathcal{L}_{n}^{\mathbb{Q}}(k+1): L_{\mathrm{GL}}^{\mu}\right]
$$

where $\mu$ runs over all partitions obtained by removing a single node from $\lambda$.

Proposition 7.3 (i) The multiplicities of the $\operatorname{Sp}(2 g, \mathbb{Q})$-irreducible representation $[k]$ in $\mathfrak{h}_{g, 1}^{\mathbb{Q}}(k)$ and $\mathcal{C}_{2 g}^{\mathbb{Q}}(k)$ are given by

$$
\left[\mathfrak{h}_{g, 1}^{\mathbb{Q}}(k): L_{\mathrm{Sp}}^{[k]}\right]=\left\{\begin{array}{ll}
1 & \text { if } k \text { odd }, \\
0 & \text { if } k \text { even, }
\end{array} \quad\left[\mathcal{C}_{2 g}^{\mathbb{Q}}(k): L_{\mathrm{Sp}}^{[k]}\right]=1 .\right.
$$

(ii) The multiplicities of the $\operatorname{Sp}(2 g, \mathbb{Q})$-irreducible representation $\left[1^{k}\right]$ in $\mathfrak{h}_{g, 1}^{\mathbb{Q}}(k)$ and $\mathcal{C}_{2 g}^{\mathbb{Q}}(k)$ are given by

$$
\left[\mathfrak{h}_{g, 1}^{\mathbb{Q}}(k): L_{\mathrm{Sp}}^{\left[1^{k}\right]}\right]=\left\{\begin{array}{ll}
1 & \text { if } k \equiv 1,2(\bmod 4), \\
0 & \text { if otherwise, }
\end{array} \quad\left[\mathcal{C}_{2 g}^{\mathbb{Q}}(k): L_{\mathrm{Sp}}^{\left[1^{k}\right]}\right]= \begin{cases}1 & \text { if } k \text { odd }, \\
0 & \text { if } k \text { even }\end{cases}\right.
$$

Proof We will use irreducible decompositions of the restriction $\operatorname{Res}_{\mathrm{Sp}}^{\mathrm{GL}}$ (see Theorem 4.5) and Pier's rule (see Theorem 4.4).

(i) If $\operatorname{Res}_{\mathrm{Sp}(2 g, \mathbb{Q})}^{\mathrm{GL}(2 g, \mathbb{Q})} L_{\mathrm{GL}}^{(\lambda)}$ has an Sp-irreducible component $L_{\mathrm{Sp}}^{[k]}$, then a partition $\lambda$ is either $\lambda=(k+1,1)$ or $\left(k, 1^{2}\right)$. We have

$$
\begin{aligned}
{\left[H_{\mathbb{Q}} \otimes_{\mathbb{Q}} \mathcal{L}_{2 g}^{\mathbb{Q}}(k+1): L_{\mathrm{GL}}^{(k+1,1)}\right] } & =\left[\mathcal{L}_{2 g}^{\mathbb{Q}}(k+1): L_{\mathrm{GL}}^{(k+1)}\right]+\left[\mathcal{L}_{2 g}^{\mathbb{Q}}(k+1): L_{\mathrm{GL}}^{(k, 1)}\right]=1, \\
{\left[\mathcal{L}_{2 g}^{\mathbb{Q}}(k+2): L_{\mathrm{GL}}^{(k+1,1)}\right] } & =1, \\
{\left[H_{\mathbb{Q}} \otimes_{\mathbb{Q}} \mathcal{L}_{2 g}^{\mathbb{Q}}(k+1): L_{\mathrm{GL}}^{\left(k, 1^{2}\right)}\right] } & =\left[\mathcal{L}_{2 g}^{\mathbb{Q}}(k+1): L_{\mathrm{GL}}^{\left(k-1,1^{2}\right)}\right]+\left[\mathcal{L}_{2 g}^{\mathbb{Q}}(k+1): L_{\mathrm{GL}}^{(k, 1)}\right], \\
& = \begin{cases}\frac{k-2}{2}+1 & \text { if } k \text { even, } \\
\frac{k-1}{2}+1 & \text { if } k \text { odd, }\end{cases} \\
{\left[\mathcal{L}_{2 g}^{\mathbb{Q}}(k+2): L_{\mathrm{GL}}^{\left(k, 1^{2}\right)}\right] } & = \begin{cases}\frac{k}{2} & \text { if } k \text { even, } \\
\frac{k-1}{2} & \text { if } k \text { odd, },\end{cases} \\
{\left[\mathcal{C}_{2 g}^{\mathbb{Q}}(k): L_{\mathrm{Sp}}^{[k]}\right] } & =\left[\mathcal{C}_{2 g}^{\mathbb{Q}}(k): L_{\mathrm{GL}}^{(k)}\right]=1 .
\end{aligned}
$$

Thus we obtain the claim. 
(ii) If $\operatorname{Res}_{\mathrm{Sp}(2 g, \mathbb{Q})}^{\mathrm{GL}(2 g, \mathbb{Q})} L_{\mathrm{GL}}^{(\lambda)}$ has an Sp-irreducible component $L_{\mathrm{Sp}}^{\left[1^{k}\right]}$, then a partition $\lambda$ is either $\lambda=\left(2^{2}, 1^{k-2}\right),\left(2,1^{k}\right)$ or $\left(1^{k+2}\right)$. We have

$$
\begin{aligned}
{\left[H_{\mathbb{Q}} \otimes \mathbb{Q} \mathcal{L}_{2 g}^{\mathbb{Q}}(k+1): L_{\mathrm{GL}}^{\left(1^{k+2}\right)}\right] } & =\left[\mathcal{L}_{2 g}^{\mathbb{Q}}(k+1): L_{\mathrm{GL}}^{\left(1^{k+1}\right)}\right]=0, \\
{\left[\mathcal{L}_{2 g}^{\mathbb{Q}}(k+2): L_{\mathrm{GL}}^{\left(1^{k+2}\right)}\right] } & =0, \\
{\left[H_{\mathbb{Q}} \otimes \mathcal{Q}_{2 g}^{\mathbb{Q}}(k+1): L_{\mathrm{GL}}^{\left(2,1^{k}\right)}\right] } & =\left[\mathcal{L}_{2 g}^{\mathbb{Q}}(k+1): L_{\mathrm{GL}}^{\left(1^{k+1}\right)}\right]+\left[\mathcal{L}_{2 g}^{\mathbb{Q}}(k+1): L_{\mathrm{GL}}^{\left(2,1^{k-1}\right)}\right]=1, \\
{\left[\mathcal{L}_{2 g}^{\mathbb{Q}}(k+2): L_{\mathrm{GL}}^{\left(2,1^{k}\right)}\right] } & =1, \\
{\left[\mathcal{C}_{2 g}^{\mathbb{Q}}(k): L_{\mathrm{Sp}}^{\left[1^{k}\right.}\right] } & =\left[\mathcal{C}_{2 g}^{\mathbb{Q}}(k): L_{\mathrm{GL}}^{\left(1^{k}\right)}\right]= \begin{cases}1 & \text { if } k \text { odd }, \\
0 & \text { if } k \text { even. } .\end{cases}
\end{aligned}
$$

Suppose $k \equiv 1,3(\bmod 4)$. Then

$$
\begin{aligned}
{\left[H_{\mathbb{Q}} \otimes_{\mathbb{Q}} \mathcal{L}_{2 g}^{\mathbb{Q}}(k+1): L_{\mathrm{GL}}^{\left(2^{2}, 1^{k-2}\right)}\right] } & \\
& =\left[\mathcal{L}_{2 g}^{\mathbb{Q}}(k+1): L_{\mathrm{GL}}^{\left(2^{2}, 1^{k-3}\right)}\right]+\left[\mathcal{L}_{2 g}^{\mathbb{Q}}(k+1): L_{\mathrm{GL}}^{\left(2,1^{k-1}\right)}\right], \\
& = \begin{cases}\frac{k-1}{2} & \text { if } k \equiv 3(\bmod 4), \\
\frac{k+1}{2} & \text { if } k \equiv 1(\bmod 4),\end{cases}
\end{aligned}
$$$$
\left[\mathcal{L}_{2 g}^{\mathbb{Q}}(k+2): L_{\mathrm{GL}}^{\left(2^{2}, 1^{k-2}\right)}\right]=\frac{k-1}{2} .
$$

Suppose $k \equiv 0,2(\bmod 4)$. Then

$\left[H_{\mathbb{Q}} \otimes_{\mathbb{Q}} \mathcal{L}_{2 g}^{\mathbb{Q}}(k+1): L_{\mathrm{GL}}^{\left(2^{2}, 1^{k-2}\right)}\right]$

$$
=\left[\mathcal{L}_{2 g}^{\mathbb{Q}}(k+1): L_{\mathrm{GL}}^{\left(2^{2}, 1^{k-3}\right)}\right]+\left[\mathcal{L}_{2 g}^{\mathbb{Q}}(k+1): L_{\mathrm{GL}}^{\left(2,1^{k-1}\right)}\right]=\frac{k}{2},
$$

$\left[\mathcal{L}_{2 g}^{\mathbb{Q}}(k+2): L_{\mathrm{GL}}^{\left(2^{2}, 1^{k-2}\right)}\right]= \begin{cases}\frac{k-2}{2} & \text { if } k \equiv 2(\bmod 4), \\ \frac{k}{2} & \text { if } k \equiv 0(\bmod 4) .\end{cases}$

Hence we obtain the claim.

Remark 7.4 By the argument above, the $\mathrm{Sp}$-irreducible component $\left[1^{k}\right]_{\text {Sp }}$ appears in the restriction of the GL-irreducible component $\left(2^{2}, 1^{k-2}\right)_{\mathrm{GL}}$.

Remark 7.5 Our calculation above gives a combinatorial description of the GL (and Sp) irreducible decomposition of $\mathfrak{h}_{g, 1}^{\mathbb{Q}}$ obtained by Kontsevich in $[17 ; 18]$.

Remark 7.6 In [24], Nakamura and Tsunogai completely calculated Sp-irreducible decompositions of $\mathfrak{h}_{g, 1}^{\mathbb{Q}}(k)$ for $1 \leq k \leq 15$. In their table, we can check that $\mathrm{Sp}-$ irreducible components $\left[1^{k}\right]$ have multiplicity one for $k=5,9,13$ and $k=6,10,14$. 


\subsection{Descriptions of maximal vectors}

To give an explicit description of maximal vectors, we use an $(i, j)$-expansion operator $D_{i j}: H_{\mathbb{Q}}^{\otimes k} \rightarrow H_{\mathbb{Q}}^{\otimes(k+2)}$ defined by

$\left(v_{1} \otimes v_{2} \otimes \cdots \otimes v_{k}\right) \cdot D_{i j}:=\sum_{r=1}^{2 g} v_{1} \otimes \cdots \otimes v_{i-1} \otimes e_{r} \otimes v_{i} \otimes \cdots \otimes v_{j-2} \otimes e_{r}^{*} \otimes v_{j-1} \otimes \cdots \otimes v_{k}$

for $1 \leq i<j \leq k+2$. Using this, we obtain several maximal vectors satisfying the condition of Proposition 7.1. First we consider a maximal vector that defines the Morita obstruction $[k]$ in $\operatorname{Coker}\left(\operatorname{Im} \tau_{k, \mathbb{Q}}^{\mathcal{M}}\right)$.

Theorem 7.7 (Morita and Nakamura) Let $k$ be an odd integer such that $k \geq 3$. Suppose $g \geq k+2$. An element

$$
\begin{aligned}
\varphi_{[k]} & :=\left(\omega \otimes e_{1}^{\otimes k}\right) \cdot \theta_{P} \cdot\left(1+\sigma_{k+2}+\cdots+\sigma_{k+2}^{k+1}\right) \\
& =2\left(\sum_{i=1}^{k+1} \sum_{r=1}^{k-i+2}(-1)^{r-1}{ }_{k} C_{r-1}\left(e_{1}^{\otimes k}\right) \cdot D_{i, i+r}\right)
\end{aligned}
$$

is a maximal vector with highest weight $[k]$ in $\mathfrak{h}_{g, 1}^{\mathbb{Q}}(k)$. Moreover this gives a unique irreducible component of $[k]$ in $\operatorname{Coker} \tau_{k, \mathbb{Q}}^{\mathcal{M}}$.

This fact was originally showed by Morita and Nakamura. More precisely, Morita [22] showed that $[k]$ appears in $\mathfrak{h}_{g, 1}^{\mathbb{Q}}(k)$ for odd $k \geq 3$ with multiplicity at least one, using the Morita trace map. Nakamura showed that the multiplicity of $[k]$ in $\mathfrak{h}_{g, 1}^{\mathbb{Q}}(k)$ for odd $k \geq 3$ is exactly one, and determined the maximal vector with highest weight $[k]$ in his unpublished work.

Second we consider a maximal vector that defines the $\operatorname{Sp}(2 g, \mathbb{Q})$-module with highest weight $\left[1^{k}\right]$ in $\operatorname{Coker}\left(\operatorname{Im} \tau_{k, \mathbb{Q}}^{\mathcal{M}}\right)$ for $k \equiv 1(\bmod 4)$ and $k \geq 5$.

Theorem 7.8 Suppose $k \equiv 1(\bmod 4), k \geq 5$ and $g \geq k+2$. An element

$$
\begin{aligned}
\varphi_{\left[1^{k}\right]} & :=\left(\omega \otimes\left(e_{1} \wedge \cdots \wedge e_{k}\right)\right) \cdot \theta_{P} \cdot\left(1+\sigma_{k+2}+\cdots+\sigma_{k+2}^{k+1}\right) \\
& =2\left(\sum_{i=1}^{k+1} \sum_{r=1}^{k-i+2}(-1)^{\delta_{r} \equiv 2,3(\bmod 4)} \frac{k-1}{2} C_{\left\lfloor\frac{r-1}{2}\right\rfloor}\left(e_{1} \wedge \cdots \wedge e_{k}\right) \cdot D_{i, i+r}\right)
\end{aligned}
$$

is a maximal vector with highest weight $\left[1^{k}\right]$ in $\mathfrak{h}_{g, 1}^{\mathbb{Q}}(k)$. Moreover this gives a unique irreducible component of $\left[1^{k}\right]$ in Coker $\tau_{k, \mathbb{Q}}^{\mathcal{M}}$. 


\subsection{Proofs of main theorems}

We will give proofs of Theorem 7.7 and Theorem 7.8. But, since our proof for Theorem 7.7 is easier than that of Theorem 7.8, we omit the details for Theorem 7.7.

7.4.1 Proof of Theorem 7.8 Step 1 For $p \equiv 2(\bmod 4)$, we prove

$$
\begin{aligned}
\left(e_{1} \wedge \cdots \wedge e_{k}\right) D_{12}\left(1-s_{2}\right)(1 & \left.-s_{3} s_{2}\right) \cdots\left(1-s_{p} \cdots s_{3} s_{2}\right) \\
& =\sum_{j=1}^{p}(-1)^{\delta_{j \equiv 2,3(\bmod 4)}} \frac{{ }_{\frac{p-2}{2}} C_{\left\lfloor\frac{j-1}{2}\right\rfloor}\left(e_{1} \wedge \cdots \wedge e_{k}\right) D_{1,1+j}}{}
\end{aligned}
$$

by the induction on $r$.

Indeed, if $p=2$, both sides of the formula above coincide with $\left(e_{1} \wedge \cdots \wedge e_{k}\right)\left(D_{12}-D_{13}\right)$. Suppose $p>2$ and $p+4 \leq k+1$. For simplicity we denote $\left(e_{1} \wedge \cdots \wedge e_{k}\right) D_{i j}$ by $D_{i, j}^{\mathrm{sgn}}$. We have:

$$
\begin{aligned}
& D_{1,1+j}^{\text {sgn }}\left(1-s_{p+1} \cdots s_{2}\right)\left(1-s_{p+2} \cdots s_{2}\right)\left(1-s_{p+3} \cdots s_{2}\right)\left(1-s_{p+4} \cdots s_{2}\right) \\
& =\left(D_{1,1+j}^{\mathrm{sgn}}-(-1)^{p+1} D_{1,2+j}^{\mathrm{sgn}}\right)\left(1-s_{p+2} \cdots s_{2}\right)\left(1-s_{p+3} \cdots s_{2}\right)\left(1-s_{p+4} \cdots s_{2}\right) \\
& \stackrel{p \text { even }}{=}\left(D_{1,1+j}^{\mathrm{sgn}}+D_{1,2+j}^{\mathrm{sgn}}\right)\left(1-s_{p+2} \cdots s_{2}\right)\left(1-s_{p+3} \cdots s_{2}\right)\left(1-s_{p+4} \cdots s_{2}\right) \\
& =\left(D_{1,1+j}^{\mathrm{sgn}}+D_{1,2+j}^{\mathrm{sgn}}\right. \\
& \left.-(-1)^{p+2} D_{1,2+j}^{\mathrm{sgn}}-(-1)^{p+2} D_{1,3+j}^{\mathrm{sgn}}\right)\left(1-s_{p+3} \cdots s_{2}\right)\left(1-s_{p+4} \cdots s_{2}\right) \\
& \stackrel{p \text { even }}{=}\left(D_{1,1+j}^{\mathrm{sgn}}-D_{1,3+j}^{\mathrm{sgn}}\right)\left(1-s_{p+3} \cdots s_{2}\right)\left(1-s_{p+4} \cdots s_{2}\right)
\end{aligned}
$$$$
=\left(D_{1,1+j}^{\mathrm{sgn}}-D_{1,3+j}^{\mathrm{sgn}}-(-1)^{p+3} D_{1,2+j}^{\mathrm{sgn}}+(-1)^{p+3} D_{1,4+j}^{\mathrm{sgn}}\right)\left(1-s_{p+4} \cdots s_{2}\right)
$$$$
\stackrel{p \text { even }}{=}\left(D_{1,1+j}^{\mathrm{sgn}}+D_{1,2+j}^{\mathrm{sgn}}-D_{1,3+j}^{\mathrm{sgn}}-D_{1,4+j}^{\mathrm{sgn}}\right)\left(1-s_{p+4} \cdots s_{2}\right)
$$

$$
\begin{aligned}
=D_{1,1+j}^{\mathrm{sgn}}+D_{1,2+j}^{\mathrm{sgn}}-D_{1,3+j}^{\mathrm{sgn}} & -D_{1,4+j}^{\mathrm{sgn}} \\
& -(-1)^{p+4}\left(D_{1,2+j}^{\mathrm{sgn}}+D_{1,3+j}^{\mathrm{sgn}}-D_{1,4+j}^{\mathrm{sgn}}-D_{1,5+j}^{\mathrm{sgn}}\right)
\end{aligned}
$$

$$
\stackrel{p \text { even }}{=} D_{1,1+j}^{\mathrm{sgn}}-2 D_{1,3+j}^{\mathrm{sgn}}+D_{1,5+j}^{\mathrm{sgn}} \text {. }
$$

Thus the action of $\left(1-s_{p+1} \cdots s_{2}\right)\left(1-s_{p+2} \cdots s_{2}\right)\left(1-s_{p+3} \cdots s_{2}\right)\left(1-s_{p+4} \cdots s_{2}\right)$ on

$$
\sum_{j=1}^{p}(-1)^{\delta_{j \equiv 2,3(\bmod 4)}} \frac{p-2}{2} C_{\left\lfloor\frac{j-1}{2}\right\rfloor} D_{1,1+j}^{\text {sgn }}
$$

is obtained in the following way:

$$
\sum_{j=1}^{p}(-1)^{\delta_{j} \equiv 2,3(\bmod 4)} \frac{p-2}{2} C_{\left\lfloor\frac{j-1}{2}\right\rfloor}\left(D_{1,1+j}^{\mathrm{sgn}}-2 D_{1,3+j}^{\mathrm{sgn}}+D_{1,5+j}^{\mathrm{sgn}}\right)
$$




$$
\begin{aligned}
& =\sum_{j=5}^{p}\left\{(-1)^{\delta_{j \equiv 2,3(\bmod 4)}} \frac{p-2}{2} C_{\left\lfloor\frac{j-1}{2}\right\rfloor}-2(-1)^{\delta_{j \equiv 0,1(\bmod 4)}}{ }_{\frac{p-2}{2}} C_{\left\lfloor\frac{j-3}{2}\right\rfloor}\right. \\
& \left.+(-1)^{\delta_{j \equiv 2,3(\bmod 4)}}{ }_{\frac{p-2}{2}} C_{\left\lfloor\frac{j-5}{2}\right\rfloor}\right\} D_{1,1+j}^{\mathrm{sgn}} \\
& +D_{12}^{\mathrm{sgn}}-D_{13}^{\mathrm{sgn}}-\frac{p-2}{2} D_{14}^{\mathrm{sgn}}+\frac{p-2}{2} D_{15}^{\mathrm{sgn}} \\
& -2\left(D_{14}^{\mathrm{sgn}}-D_{15}^{\mathrm{sgn}}+D_{1, p+2}^{\mathrm{sgn}}-D_{1, p+3}^{\mathrm{sgn}}\right) \\
& -\frac{p-2}{2} D_{1, p+2}^{\mathrm{sgn}}+\frac{p-2}{2} D_{1, p+3}^{\mathrm{sgn}}+D_{1, p+4}^{\mathrm{sgn}}-D_{1, p+5}^{\mathrm{sgn}} \\
& =\sum_{j=1}^{p+4}(-1)^{\delta_{j \equiv 2,3(\bmod 4)}} \frac{p+2}{2} C_{\left\lfloor\frac{j-1}{2}\right\rfloor} D_{1,1+j}^{\text {sgn }} \text {. }
\end{aligned}
$$

Step 2 We have

$$
\begin{aligned}
& \left(e_{1} \wedge \cdots \wedge e_{k}\right) D_{i j} s_{k+1} \cdots s_{2} s_{1} \\
& \quad= \begin{cases}\left(e_{1} \wedge \cdots \wedge e_{k}\right)(-1)^{k-1} D_{i+1, j+1} \stackrel{k \text { odd }}{=}\left(e_{1} \wedge \cdots \wedge e_{k}\right) D_{i+1, j+1} & \text { if } j \neq k+2, \\
-\left(e_{1} \wedge \cdots \wedge e_{k}\right) D_{1, i+1} & \text { if } j=k+2,\end{cases}
\end{aligned}
$$

for $k \equiv 1(\bmod 4)$. Hence we obtain an explicit formula

$\left(\omega \otimes\left(e_{1} \wedge \cdots \wedge e_{k}\right)\right) \cdot \theta_{P} \cdot\left(1+\sigma_{k+2}+\cdots+\sigma_{k+2}^{k+1}\right)$

$$
=2 \sum_{i=1}^{k+1} \sum_{j=1}^{k-i+2}(-1)^{\delta_{j \equiv 2,3(\bmod 4)}} \frac{k-1}{2} C_{\left\lfloor\frac{j-1}{2}\right\rfloor}\left(e_{1} \wedge \cdots \wedge e_{k}\right) \cdot D_{i, i+j}
$$

In fact,

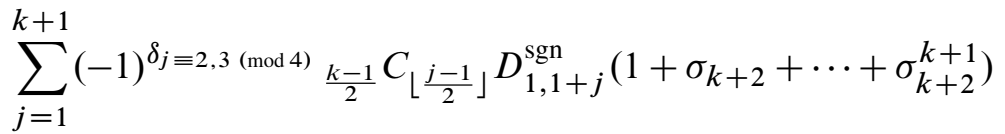

$$
\begin{aligned}
& =\sum_{j=1}^{k+1}(-1)^{\delta_{j \equiv 2,3(\bmod 4)}}{ }_{\frac{k-1}{2}} C_{\left\lfloor\frac{j-1}{2}\right\rfloor}\left(\sum_{i=1}^{k+2-j} D_{i, i+j}^{\mathrm{sgn}}-\sum_{i=1}^{j} D_{i, i+k+2-j}^{\mathrm{sgn}}\right) \\
& =\sum_{i=1}^{k+1} \sum_{j=1}^{k+2-i}(-1)^{\delta_{j \equiv 2,3(\bmod 4)}}{ }_{\frac{k-1}{2}} C_{\left\lfloor\frac{j-1}{2}\right\rfloor} D_{i, i+j}^{\mathrm{sgn}} \\
& -\sum_{i=1}^{k+1} \sum_{j=i}^{k+1}(-1)^{\delta_{j \equiv 2,3(\bmod 4)}} \frac{k-1}{2} C_{\left\lfloor\frac{j-1}{2}\right\rfloor} D_{i, i+k+2-j}^{\mathrm{sgn}}
\end{aligned}
$$




$$
\begin{aligned}
& =\sum_{i=1}^{k+1} \sum_{j=1}^{k+2-i}(-1)^{\delta_{j \equiv 2,3(\bmod 4)}}{ }_{\frac{k-1}{2}} C_{\left\lfloor\frac{j-1}{2}\right\rfloor} D_{i, i+j}^{\text {sgn }} \\
& -\sum_{i=1}^{k+1} \sum_{j=1}^{k+2-i}(-1)^{\delta_{k+2-j \equiv 2,3(\bmod 4)}} \frac{k-1}{2} C_{\left\lfloor\frac{k+1-j}{2}\right\rfloor} D_{i, i+j}^{\mathrm{sgn}} \\
& =\sum_{i=1}^{k+1} \sum_{j=1}^{k+2-i}(-1)^{\delta_{j \equiv 2,3(\bmod 4)}}{ }_{\frac{k-1}{2}} C_{\left\lfloor\frac{j-1}{2}\right\rfloor} D_{i, i+j}^{\text {sgn }} \\
& +\sum_{i=1}^{k+1} \sum_{j=1}^{k+2-i}(-1)^{\delta_{j \equiv 2,3(\bmod 4)}} \frac{k-1}{2} C_{\left\lfloor\frac{k+1-j}{2}\right\rfloor} D_{i, i+j}^{\text {sgn }} \\
& =2 \sum_{i=1}^{k+1} \sum_{j=1}^{k+2-i}(-1)^{\delta_{j \equiv 2,3(\bmod 4)}}{ }_{\frac{k-1}{2}} C_{\left\lfloor\frac{j-1}{2}\right\rfloor} D_{i, i+j}^{\text {sgn }} .
\end{aligned}
$$

Step 3 Let us consider a surjective Sp-homomorphism

$$
\operatorname{cont}_{k}: H_{\mathbb{Q}}^{\otimes(k+2)} \stackrel{\sim}{\longrightarrow} H_{\mathbb{Q}}^{*} \otimes H_{\mathbb{Q}}^{\otimes(k+1)} \rightarrow H_{\mathbb{Q}}^{\otimes k}
$$

by composing an $\mathrm{Sp}$-isomorphism

$$
H_{\mathbb{Q}}^{\otimes(k+2)} \rightarrow H_{\mathbb{Q}}^{*} \otimes H_{\mathbb{Q}}^{\otimes(k+1)}
$$

induced from $H_{\mathbb{Q}} \stackrel{\sim}{\longrightarrow} H_{\mathbb{Q}}^{*}$ given by (4) in Remark 4.3 and a contraction homomorphism defined by $e_{i}^{*} \otimes e_{j_{1}} \otimes e_{j_{2}} \otimes \cdots \otimes e_{j_{k+1}} \mapsto\left\langle e_{i}^{*}, e_{j_{1}}\right\rangle \cdot e_{j_{2}} \otimes \cdots \otimes e_{j_{k+1}}$. Then we obtain

$$
\operatorname{cont}_{k}\left(\left(e_{1} \wedge \cdots \wedge e_{k}\right) D_{i j}\right)= \begin{cases}(-2 g)\left(e_{1} \wedge \cdots \wedge e_{k}\right) & \text { if } i=1, j=2, \\ (-1)^{j-2}\left(e_{1} \wedge \cdots \wedge e_{k}\right) & \text { if } i=1, j \geq 3, \\ (-1)^{j-3}\left(e_{1} \wedge \cdots \wedge e_{k}\right) & \text { if } i=2, j \geq 3, \\ 0 & \text { if otherwise }\end{cases}
$$

To prove these formulae, let us recall that

$$
\begin{aligned}
& \left\langle e_{i}, e_{j}\right\rangle=0=\left\langle e_{i^{\prime}}, e_{j^{\prime}}\right\rangle, \\
& \left\langle e_{i}, e_{j^{\prime}}\right\rangle=\delta_{i j}=-\left\langle e_{j^{\prime}}, e_{i}\right\rangle, \\
& (1 \leq i \leq g), \quad e_{i}^{*}= \begin{cases}e_{i^{\prime}} & (1 \leq i \leq g) \\
-e_{i^{\prime}} & (g+1 \leq i \leq 2 g) .\end{cases}
\end{aligned}
$$

where $i^{\prime}:=2 g-i+1$ for each integer $1 \leq i \leq 2 g$.

Then we have

$$
\begin{aligned}
\operatorname{cont}_{k}\left(D_{12}^{\mathrm{sgn}}\right) & =\operatorname{cont}_{k}\left(\sum_{r=1}^{2 g} e_{r} \otimes e_{r}^{*} \otimes\left(e_{1} \wedge \cdots \wedge e_{k}\right)\right) \\
& =\sum_{r=1}^{2 g}\left\langle e_{r}^{*}, e_{r}\right\rangle e_{1} \wedge \cdots \wedge e_{k}=(-2 g) e_{1} \wedge \cdots \wedge e_{k}
\end{aligned}
$$


Moreover,

$$
\begin{aligned}
& \operatorname{cont}_{k}\left(D_{1 j}^{\mathrm{sgn}}\right)=\operatorname{cont}_{k}\left(\sum_{r=1}^{2 g} \sum_{\sigma \in \mathfrak{S}_{k}} \operatorname{sgn}(\sigma) e_{r} \otimes e_{\sigma(1)} \otimes e_{\sigma(2)} \otimes \cdots \otimes e_{r}^{*} \otimes \cdots \otimes e_{\sigma(k)}\right) \\
& =\sum_{r=1}^{2 g} \sum_{\sigma \in \mathfrak{S}_{k}} \operatorname{sgn}(\sigma)\left\langle e_{\sigma(1)}, e_{r}\right\rangle \otimes e_{\sigma(2)} \otimes \cdots \otimes e_{r}^{*} \otimes \cdots \otimes e_{\sigma(k)} \\
& =\sum_{\sigma \in \mathfrak{S}_{k}} \operatorname{sgn}(\sigma) e_{\sigma(2)} \otimes \cdots \otimes e_{\sigma(1)^{\prime}}^{j-2} \otimes \cdots \otimes e_{\sigma(k)} \\
& =-\sum_{\sigma \in \mathfrak{S}_{k}} \operatorname{sgn}(\sigma) e_{\sigma(2)} \otimes \cdots \otimes e_{\sigma(1)}^{j-2} \otimes \cdots \otimes e_{\sigma(k)} \\
& =-\left(e_{1} \wedge \cdots \wedge e_{k}\right) \cdot s_{1} s_{2} \cdots s_{j-3}=(-1)^{j-2} e_{1} \wedge \cdots \wedge e_{k},
\end{aligned}
$$

and similarly,

$$
\begin{aligned}
\operatorname{cont}_{k}\left(D_{2 j}^{\mathrm{sgn}}\right) & =\operatorname{cont}_{k}\left(\sum_{r=1}^{2 g} \sum_{\sigma \in \mathfrak{S}_{k}} \operatorname{sgn}(\sigma) e_{\sigma(1)} \otimes e_{r} \otimes e_{\sigma(2)} \otimes \cdots \otimes e_{r}^{*} \otimes \cdots \otimes e_{\sigma(k)}\right. \\
& =\sum_{r=1}^{2 g} \sum_{\sigma \in \mathfrak{S}_{k}} \operatorname{sgn}(\sigma)\left\langle e_{r}, e_{\sigma(1)}\right\rangle \otimes e_{\sigma(2)} \otimes \cdots \otimes e_{r}^{*} \otimes \cdots \otimes e_{\sigma(k)}{ }^{-2} \\
& =\sum_{\sigma \in \mathfrak{S}_{k}} \operatorname{sgn}(\sigma) e_{\sigma(2)} \otimes \cdots \otimes e_{\sigma(1)^{\prime}}^{*} \otimes \cdots \otimes e_{\sigma(k)} \\
= & \sum_{\sigma \in \mathfrak{S}_{k}} \operatorname{sgn}(\sigma) e_{\sigma(2)} \otimes \cdots \otimes e_{\sigma(1)}^{j-V^{2}} \otimes \cdots \otimes e_{\sigma(k)} \\
= & \left(e_{1} \wedge \cdots \wedge e_{k}\right) \cdot s_{1} s_{2} \cdots s_{j-3}=(-1)^{j-3} e_{1} \wedge \cdots \wedge e_{k} .
\end{aligned}
$$

For $i \geq 3$, because of $g>k$, it is clear that $\operatorname{cont}_{k}\left(\left(e_{1} \wedge \cdots \wedge e_{k}\right) D_{i j}\right)=0$.

Step 4 We obtain $c_{k}\left(\varphi_{\left[1^{k}\right]}\right) \neq 0$.

Indeed, for the natural surjection pr: $H_{\mathbb{Q}}^{\otimes k} \rightarrow \mathcal{C}_{2 g}^{\mathbb{Q}}(k)$, we have

$$
c\left(\varphi_{\left[1^{k}\right]}\right)=2\left(\begin{array}{l}
\sum_{j=1}^{k+1}(-1)^{\delta_{j \equiv 2,3(\bmod 4)}}{ }_{\frac{k-1}{2}} C_{\left\lfloor\frac{j-1}{2}\right\rfloor} c_{k}\left(e_{1} \wedge \cdots \wedge e_{k} D_{1,1+j}\right) \\
+\sum_{j=1}^{k}(-1)^{\delta_{j \equiv 2,3(\bmod 4)}}{ }_{\frac{k-1}{2}} C_{\left\lfloor\frac{j-1}{2}\right\rfloor} c_{k}\left(e_{1} \wedge \cdots \wedge e_{k} D_{2,2+j}\right)
\end{array}\right)
$$




$$
\begin{aligned}
& =2\left(\begin{array}{l}
-2 g+\sum_{j=2}^{k+1}(-1)^{\delta_{j \equiv 2,3(\bmod 4)}}{ }_{\frac{k-1}{2}} C_{\left\lfloor\frac{j-1}{2}\right\rfloor}(-1)^{j-1} \\
+\sum_{j=1}^{k}(-1)^{\delta_{j \equiv 2,3(\bmod 4)}}{ }_{\frac{k-1}{2}} C_{\left\lfloor\frac{j-1}{2}\right\rfloor}(-1)^{j-1}
\end{array}\right) \operatorname{pr}\left(e_{1} \wedge \cdots \wedge e_{k}\right) \\
= & 2\left(-2 g+2+2 \sum_{j=2}^{k}(-1)^{j-1+\delta_{j \equiv 2,3(\bmod 4)}} \frac{k-1}{2} C_{\left\lfloor\frac{j-1}{2}\right\rfloor}\right) \operatorname{pr}\left(e_{1} \wedge \cdots \wedge e_{k}\right) \\
= & 2\left(-2 g-2+2 \sum_{j=1}^{k+1}(-1)^{j-1+\delta_{j \equiv 2,3(\bmod 4)}} \frac{k-1}{2} C_{\left\lfloor\frac{j-1}{2}\right\rfloor}\right) \operatorname{pr}\left(e_{1} \wedge \cdots \wedge e_{k}\right) .
\end{aligned}
$$

Here, we claim that

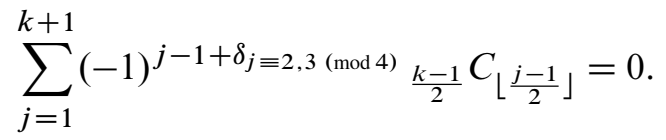

In fact, by setting $k=4 K+1$, we have

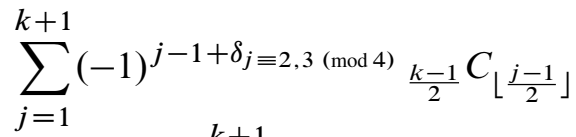

$$
\begin{aligned}
& =\sum_{j=1}^{k+1}(-1)^{\delta_{j \equiv 0,3(\bmod 4)}} \frac{k-1}{2} C_{\left\lfloor\frac{j-1}{2}\right\rfloor} \\
& =\sum_{\substack{1 \leq j \leq k+1 \\
j \text { odd }}}(-1)^{\delta_{j \equiv 3(\bmod 4)}} \frac{k-1}{2} C_{\left\lfloor\frac{j-1}{2}\right\rfloor}+\sum_{\substack{1 \leq j \leq k+1 \\
j \text { even }}}(-1)^{\delta_{j \equiv 0(\bmod 4)} \frac{k-1}{2} C_{\left\lfloor\frac{j-1}{2}\right\rfloor}} \\
& =\sum_{p=0}^{2 K}(-1)^{\delta_{p \equiv 1(\bmod 2)}} 2{ }_{K} C_{p}+\sum_{q=1}^{2 K+1}(-1)^{\delta_{q \equiv 0(\bmod 2)}{ }_{2 K} C_{q-1}} \\
& =2 \sum_{p=0}^{2 K}(-1)^{\delta_{p \equiv 1(\bmod 2)}}{ }_{2 K} C_{p}=2(1-1)^{2 K}=0 \text {. }
\end{aligned}
$$

Hence, we conclude $c_{k}\left(\varphi_{\left[1^{k}\right]}\right)=-4(g+1) \operatorname{pr}\left(e_{1} \wedge \cdots \wedge e_{k}\right)$.

Since $\left[L^{\left[1^{k}\right]}: H_{\mathbb{Q}}^{\otimes k}\right]=\left[L^{\left[1^{k}\right]}: \mathcal{C}_{2 g}^{\mathbb{Q}}(k)\right]=1$ and $e_{1} \wedge \cdots \wedge e_{k}$ is a maximal vector with highest weight $\left(1^{k}\right)$ of $H_{\mathbb{Q}}^{\otimes k}$, we have $\operatorname{pr}\left(e_{1} \wedge \cdots \wedge e_{k}\right) \neq 0$.

Step 5 By Proposition 7.1 and Proposition 7.3, the maximal vector $\varphi_{\left[1^{k}\right]}$ gives a unique irreducible component of $\left[1^{k}\right]$ in $\operatorname{Coker} \tau_{k, \mathbb{Q}}^{\mathcal{M}}$.

This completes the proof of Theorem 7.8. 
7.4.2 Outline of proof of Theorem 7.7 To begin with, we can show $\left(e_{1}^{\otimes k} D_{12}\right)\left(1-s_{2}\right)\left(1-s_{3} s_{2}\right) \cdots\left(1-s_{r} \cdots s_{3} s_{2}\right)=\sum_{j=1}^{r}(-1)^{j-1}{ }_{r-1} C_{j-1}\left(e_{1}^{\otimes k}\right) D_{1,1+j}$ by using induction on $r$. Secondly, we have

$$
\left(e_{1}^{\otimes k} D_{i j}\right) s_{k+1} s_{k} \cdots s_{2} s_{1}= \begin{cases}e_{1}^{\otimes k} D_{i+1, j+1} & \text { if } j \neq k+2 \\ -e_{1}^{\otimes k} D_{1, i+1} & \text { if } j=k+2 .\end{cases}
$$

Hence we get an explicit formula

$\left(\omega \otimes e_{1}^{\otimes k}\right) \cdot \theta_{P} \cdot\left(1+\sigma_{k+2}+\cdots+\sigma_{k+2}^{k+1}\right)=\sum_{i=1}^{k+1} \sum_{r=1}^{k-i+2}(-1)^{r-1} C_{r-1}\left(e_{1}^{\otimes k}\right) \cdot D_{i, i+r}$.

Thirdly, we have

$$
\operatorname{cont}_{k}\left(e_{1}^{\otimes k} D_{i j}\right)= \begin{cases}(-2 g)\left(e_{1}^{\otimes k}\right) & \text { if } i=1, j=2, \\ -\left(e_{1}^{\otimes k}\right) & \text { if } i=1, j \geq 3, \\ \left(e_{1}^{\otimes k}\right) & \text { if } i=2, j \geq 3, \\ 0 & \text { otherwise, }\end{cases}
$$

and $\operatorname{pr}\left(e_{1}^{\otimes k}\right) \neq 0$. Thus we obtain

$$
\begin{aligned}
c_{k}\left(\varphi_{[k]}\right) & =\sum_{j=1}^{k+1}(-1)^{j-1}{ }_{k} C_{j-1} c_{k}\left(e_{1}^{\otimes k} D_{1 j}\right)+\sum_{j=1}^{k}(-1)^{j-1}{ }_{k} C_{j-1} c_{k}\left(e_{1}^{\otimes k} D_{2 j}\right) \\
& =\left(-2 g-\sum_{j=2}^{k+1}(-1)^{j-1}{ }_{k} C_{j-1}+\sum_{j=1}^{k}(-1)^{j-1}{ }_{k} C_{j-1}\right) \operatorname{pr}\left(e_{1}^{\otimes k}\right) \\
& =\left(-2 g+(-1)^{k+1}+\sum_{j=2}^{k}\left\{(-1)^{j}{ }_{k} C_{j}+(-1)^{j-1}{ }_{k} C_{j-1}\right\}+1\right) \operatorname{pr}\left(e_{1}^{\otimes k}\right) \\
& =(2-2 g) \operatorname{pr}\left(e_{1}^{\otimes k}\right) \neq 0 .
\end{aligned}
$$

Therefore, by Proposition 7.1 and Proposition 7.3, the maximal vector $\varphi_{[k]}$ gives a unique irreducible component of $[k]$ in $\operatorname{Coker} \tau_{k, \mathbb{Q}}^{\mathcal{M}}$.

This completes the proof of Theorem 7.7.

\subsection{Problems for the Johnson cokernels}

Finally, we conclude by suggesting a problem for the Johnson cokernels of the mapping class group. 
By observing the table of $\operatorname{Coker}\left(\tau_{k, \mathbb{Q}}^{\mathcal{M}}\right)$ for $1 \leq k \leq 4$ in Section 3.3, we see that $\operatorname{Coker}\left(\tau_{k, \mathbb{Q}}^{\mathcal{M}}\right) \cong \operatorname{Im}\left(c_{k}\right)$ for $1 \leq k \leq 4$ as an $\operatorname{Sp}(2 g, \mathbb{Q})$-module, where $c_{k}: \mathfrak{h}_{g, 1}^{\mathbb{Q}}(k) \rightarrow$ $\mathcal{C}_{2 g}^{\mathbb{Q}}(k)$ is an $\operatorname{Sp}(2 g, \mathbb{Q})$-equivariant homomorphism defined in Section 7.1.

In general the cokernel $\operatorname{Coker}\left(\tau_{k, \mathbb{Q}}^{\mathcal{M}}\right)$ is not isomorphic to $\operatorname{Im}\left(c_{k}\right)$ for $k \geq 6$. In fact, for $k=6$ according to the description in [23], the $\operatorname{Sp}$-invariant part of $\mathfrak{h}_{g, 1}^{\mathbb{Q}}(6) / \operatorname{Im}\left(\tau_{6, \mathbb{Q}}^{\mathcal{M}}\right)$ is $\mathbb{Q}^{\oplus 3}$. On the other hand, that of $\mathcal{C}_{2 g}^{\mathbb{Q}}(6)$ is $\mathbb{Q}^{\oplus 2}$. Hence we can not detect all of the Sp-invariant part of $\mathfrak{h}_{g, 1}^{\mathbb{Q}}(6)$ using the map $c_{6}$. We have heard from Morita about these facts in a thoughtful e-mail.

Here we suggest a problem to determine the $S p$-component of $\mathfrak{h}_{g, 1}^{\mathbb{Q}}(k)$ that can be detected by the map $c_{k}$. Namely:

Problem 7.9 For any $k \geq 1$, determine the image $\operatorname{Im}\left(c_{k}\right)$ of $c_{k}$.

Let us consider a sequence of Sp-submodules of $\mathfrak{h}_{g, 1}^{\mathbb{Q}}$,

$$
\operatorname{Im}\left(\tau_{k, \mathbb{Q}}^{\mathcal{M}}\right) \subset \operatorname{Ker}\left(c_{k}\right) \subset \mathfrak{h}_{g, 1}^{\mathbb{Q}},
$$

for each $k \geq 2$. Problem 7.9 is equivalent to a problem to determine the $\mathrm{Sp}$-module structure of the quotient $\mathfrak{h}_{g, 1}^{\mathbb{Q}} / \operatorname{Ker}\left(c_{k}\right)$. We remark that from the description in [23] as above, for $k=6$, an irreducible module [0] appears in $\operatorname{Ker}\left(c_{6}\right) / \operatorname{Im}\left(\tau_{6, \mathbb{Q}}^{\mathcal{M}}\right)$ with multiplicity at least one. (Morita told us this fact in his e-mail to us.) This shows $\operatorname{Im}\left(\tau_{k, \mathbb{Q}}^{\mathcal{M}}\right) \neq \operatorname{Ker}\left(c_{k}\right)$ in general.

Let $\left(\mathfrak{h}_{g, 1}^{\mathbb{Q}}\right)^{\text {ab }}$ be the abelianization of $\mathfrak{h}_{g, 1}^{\mathbb{Q}}$ as a Lie algebra, and $\left[\mathfrak{h}_{g, 1}^{\mathbb{Q}}, \mathfrak{h}_{g, 1}^{\mathbb{Q}}\right]$ the kernel of the abelianization $\mathfrak{h}_{g, 1}^{\mathbb{Q}} \rightarrow\left(\mathfrak{h}_{g, 1}^{\mathbb{Q}}\right)^{\mathrm{ab}}$. We write

$$
\left[\mathfrak{h}_{g, 1}^{\mathbb{Q}}, \mathfrak{h}_{g, 1}^{\mathbb{Q}}\right](k)
$$

for the degree $k$ part of $\left[\mathfrak{h}_{g, 1}^{\mathbb{Q}}, \mathfrak{h}_{g, 1}^{\mathbb{Q}}\right]$. It is still open problem to determine the $\mathrm{Sp}$-module structure of $\left(\mathfrak{h}_{g, 1}^{\mathbb{Q}}\right)^{\mathrm{ab}}$. From Hain's result (see Theorem 3.5) we have

$$
\operatorname{Im}\left(\tau_{k, \mathbb{Q}}^{\mathcal{M}}\right) \subset\left[\mathfrak{h}_{g, 1}^{\mathbb{Q}}, \mathfrak{h}_{g, 1}^{\mathbb{Q}}\right](k) \subset \mathfrak{h}_{g, 1}^{\mathbb{Q}}
$$

for each $k \geq 2$. In [23], Morita constructed a surjective Lie algebra homomorphism

$$
\tau_{1, \mathbb{Q}}^{\mathcal{M}} \oplus \bigoplus_{k \geq 1} \operatorname{Tr}_{2 k+1}: \mathfrak{h}_{g, 1}^{\mathbb{Q}} \rightarrow \Lambda^{3} H_{\mathbb{Q}} \oplus \bigoplus_{k \geq 1} S^{2 k+1} H_{\mathbb{Q}}
$$

using the Morita trace maps $\operatorname{Tr}_{2 k+1}$, where the target is considered as an abelian Lie algebra. Hence, the Morita obstructions can be detected by $\mathfrak{h}_{g, 1}^{\mathbb{Q}}(k) / \operatorname{Ker}\left(c_{k}\right)$ and $\left(\mathfrak{h}_{g, 1}^{\mathbb{Q}}\right)^{\mathrm{ab}}$. Recently, in [4], J Conant, M Kassabov and K Vogtmann announced there are new series in $\left(\mathfrak{h}_{g, 1}^{\mathbb{Q}}\right)^{\mathrm{ab}}$ other than the Morita obstructions.

Then we have a problem: 
Problem 7.10 Does there exist an irreducible $\mathrm{Sp}$-module $L \subset \operatorname{Ker}\left(c_{k}\right)$ such that $L \not \subset$ $\left[\mathfrak{h}_{g, 1}^{\mathbb{Q}}, \mathfrak{h}_{g, 1}^{\mathbb{Q}}\right](k)$ ? For example, clarify whether or not the Conant-Kassabov-Vogtmann obstruction is contained in $\operatorname{Ker}\left(c_{k}\right)$.

\section{Acknowledgements}

Both authors would like to thank Professor Shigeyuki Morita and Takuya Sakasai for valuable discussions about our results and related topics and sincere encouragement for our research. They would also like to thank J Conant and M Kassabov for the discussion about their recent works.

The authors are supported by JSPS Research Fellowship for Young Scientists and the Global COE program at Kyoto University.

In November 2004, at Okayama University, Professor Hiroaki Nakamura showed the second author (TS) his explicit calculation [24], and they discussed the multiplicities of $\left[1^{k}\right]$. They checked that the multiplicities of $\left[1^{k}\right]$ and in $\mathfrak{h}_{g, 1}^{\mathbb{Q}}(k)$ are exactly one for $k=5,9,13$ and $k=6,10,14$. Nakamura communicated to him the possibilities that the multiplicities of $\left[1^{k}\right]$ in $\mathfrak{h}_{g, 1}^{\mathbb{Q}}(k)$ is exactly one for general $k \geq 5$ such that $k \equiv 1(\bmod 4)$, and that they survive in the Johnson cokernels. The second author would like to thank Professor Nakamura for these suggestions which motivated him to study the mapping class group of a surface.

The first author (NE) would like to thank Kentaro Wada for his kind guidance for dealing with idempotents and the Brauer algebras. He also would like to thank Yuichiro Hoshi for his comments on the arithmetic aspects of the mapping class groups.

Both authors also would like to thank the referee for his/her careful reading and useful comments to the organization of this paper.

\section{References}

[1] S Andreadakis, On the automorphisms of free groups and free nilpotent groups, Proc. London Math. Soc. 15 (1965) 239-268 MR0188307

[2] M Asada, H Nakamura, On graded quotient modules of mapping class groups of surfaces, Israel J. Math. 90 (1995) 93-113 MR1336318

[3] N Bourbaki, Lie groups and Lie algebras, Chapters 1-3, Elements of Mathematics, Springer, Berlin (1989) MR979493

[4] J Conant, M Kassabov, K Vogtmann, Hairy graphs and the unstable homology of $\operatorname{Mod}(g, s), \operatorname{Out}\left(F_{n}\right)$ and $\operatorname{Aut}\left(F_{n}\right)$, J. Topol. 6 (2013) 119-153 MR3029423 
[5] N Enomoto, T Satoh, On the derivation algebra of the free Lie algebra and trace maps, Algebr. Geom. Topol. 11 (2011) 2861-2901 MR2846914

[6] W Fulton, J Harris, Representation theory: A first course, Graduate Texts in Mathematics 129, Springer, New York (1991) MR1153249

[7] A M Garsia, Combinatorics of the free Lie algebra and the symmetric group, from: "Analysis, et cetera", (PH Rabinowitz, E Zehnder, editors), Academic Press, Boston, MA (1990) 309-382 MR1039352

[8] R Hain, Infinitesimal presentations of the Torelli groups, J. Amer. Math. Soc. 10 (1997) 597-651 MR1431828

[9] J Hu, Dual partially harmonic tensors and Brauer-Schur-Weyl duality, Transform. Groups 15 (2010) 333-370 MR2657445

[10] J Hu, Y Yang, Some irreducible representations of Brauer's centralizer algebras, Glasg. Math. J. 46 (2004) 499-513 MR2094806

[11] D Johnson, An abelian quotient of the mapping class group $\mathcal{I}_{g}$, Math. Ann. 249 (1980) 225-242 MR579103

[12] D Johnson, The structure of the Torelli group, I: A finite set of generators for $\mathcal{I}$, Ann. of Math. 118 (1983) 423-442 MR727699

[13] D Johnson, The structure of the Torelli group, II: A characterization of the group generated by twists on bounding curves, Topology 24 (1985) 113-126 MR793178

[14] D Johnson, The structure of the Torelli group, III: The abelianization of $\mathcal{T}$, Topology 24 (1985) 127-144 MR793179

[15] A A Kljačko, Lie elements in a tensor algebra, Sibirsk. Mat. Ž. 15 (1974) 1296-1304, 1430 MR0371961

[16] K Koike, I Terada, Young-diagrammatic methods for the representation theory of the classical groups of type $B_{n}, C_{n}, D_{n}$, J. Algebra 107 (1987) 466-511 MR885807

[17] M Kontsevich, Formal (non)commutative symplectic geometry, from: "The Gel' fand Mathematical Seminars, 1990-1992”, (L Corwin, I Gel' fand, J Lepowsky, editors), Birkhäuser, Boston, MA (1993) 173-187 MR1247289

[18] M Kontsevich, Feynman diagrams and low-dimensional topology, from: "First European Congress of Mathematics, Vol. II", (A Joseph, F Mignot, F Murat, B Prum, R Rentschler, editors), Progr. Math. 120, Birkhäuser, Basel (1994) 97-121 MR1341841

[19] W Kraśkiewicz, J Weyman, Algebra of coinvariants and the action of a Coxeter element, Bayreuth. Math. Schr. (2001) 265-284 MR1867283

[20] I G Macdonald, Symmetric functions and Hall polynomials, 2nd edition, Oxford Mathematical Monographs, The Clarendon Press, New York (1995) MR1354144

[21] S Morita, Casson's invariant for homology 3-spheres and characteristic classes of surface bundles, I, Topology 28 (1989) 305-323 MR1014464 
[22] S Morita, Abelian quotients of subgroups of the mapping class group of surfaces, Duke Math. J. 70 (1993) 699-726 MR1224104

[23] S Morita, Structure of the mapping class groups of surfaces: a survey and a prospect, from: "Proceedings of the Kirbyfest", (J Hass, M Scharlemann, editors), Geom. Topol. Monogr. 2 (1999) 349-406 MR1734418

[24] H Nakamura, H Tsunogai, Atlas of pro-l mapping class groups and related topics, in preparation

[25] J Nielsen, Die Isomorphismengruppe der freien Gruppen, Math. Ann. 91 (1924) 169209 MR1512188

[26] A Ram, Characters of Brauer's centralizer algebras, Pacific J. Math. 169 (1995) 173-200 MR1346252

[27] C Reutenauer, Free Lie algebras, London Mathematical Society Monographs. New Series 7, The Clarendon Press, New York (1993) MR1231799

[28] T Satoh, On the lower central series of the IA-automorphism group of a free group, J. Pure Appl. Algebra 216 (2012) 709-717 MR2864772

[29] J-P Serre, Lie algebras and Lie groups, 2nd edition, Lecture Notes in Mathematics 1500, Springer, Berlin (1992) MR1176100

[30] E Witt, Faithful representation of Lie rings (Treue Darstellung Liescher Ringe), J. Reine Angew. Math. 177 (1937) 152-160

Department of Mathematics, Faculty of Science, Nara Women's University Kitauoyahigashi-machi, Nara city 630-8506, Japan

Department of Mathematics, Faculty of Science Division II, Tokyo University of Science Kagurazaka 1-3, Shinjuku-ku, Tokyo 1628601, Japan

enomoto@cc.nara-wu.ac.jp, takao@rs.tus.ac.jp

Received: 20 August 2012 Revised: 3 August 2013 